\title{
Network-Coded Multiple Access II: Toward Realtime Operation with Improved Performance
}

\author{
Lizhao You, Student Member, IEEE, Soung Chang Liew, Fellow, IEEE, and Lu Lu, Member, IEEE
}

\begin{abstract}
This paper presents a first real-time network-coded multiple access (NCMA) system that jointly exploits physicallayer network coding (PNC) and multi-user decoding (MUD) to boost the throughput of a wireless local area network (WLAN). NCMA is a new design paradigm for multipacket reception wireless networks, in which the access point (AP) can receive and decode several packets simultaneously transmitted by multiple users. Conventionally, multipacket reception is realized using MUD only, while the key idea of NCMA is to use PNC together with MUD to realize multipacket reception. Although the feasibility of NCMA has previously been studied by the authors, our previous NCMA prototype was a version with offline signal processing. In addition, our previous investigation left open a number of theoretical and implementation issues, the resolution of which is critical to the adoption of NCMA in real practice. The current investigation makes the following state-ofthe-art contributions toward NCMA: 1) we demonstrate a first NCMA system with integrated real-time PHY-layer and MAClayer decoding; 2) we construct a new unified framework for MAC-layer decoding that yields higher throughput with faster decoding - the faster decoding is one of the key enablers of our real-time implementation; 3) we design new PHY-layer decoding techniques that overcome the poor performance of the firstgeneration NCMA prototype at low SNR. Experimental results show that, compared with the previous NCMA prototype, our new NCMA prototype improves real-time throughput by more than $100 \%$ at medium-high SNR $(\geq 8 \mathrm{~dB})$.
\end{abstract}

\section{INTRODUCTION}

This paper presents new design techniques to move Network-Coded Multiple Access (NCMA) a step toward practicality. Specifically, we demonstrate real-time operation of NCMA for the first time using a new framework for MAClayer channel decoding. In addition, the throughput has also been improved. Furthermore, we overcome the poor low SNR performance of the previous NCMA system [1] using new PHY-layer and cross-layer interference cancellation techniques.

NCMA, first proposed in [1], is a new design paradigm for multipacket reception wireless networks. In a multipacket reception wireless local area network, the access point (AP) can receive and decode several packets simultaneously transmitted by multiple users. Fig. 1 illustrates a typical scenario. Here, two user nodes, A and B, want to send messages to the AP. To boost throughput, the two users transmit simultaneously.

Conventionally, multipacket reception is realized using the multiuser decoding (MUD) technique [2]. The key idea of NCMA is to use physical-layer network coding (PNC) decoding together with MUD to realize multimessage reception. PNC, first introduced in [3] and [4], is a technique to exploit wireless interference by turning the superimposed

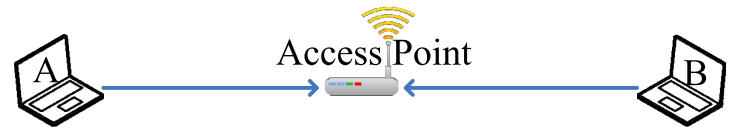

Fig. 1: Illustration of an NCMA network in which two user nodes transmit simultaneously to an access point. In general, simultaneous transmissions by more than two users are also possible.

electromagnetic waves into useful network-coded information. It has developed into a subfield of network coding with a wide following [5]. However, nearly all prior PNC systems focused on relay networks, where the relay nodes target to obtain network-coded information from the signals simultaneously transmitted by several other nodes. The process of decoding for the network-coded information based on the superimposed signals is referred to as PNC decoding. Instead of forwarding the original information of the source nodes, the relay nodes forward the network-coded information to destination nodes, where self-information or side information obtained by overhearing [6] is used to extract the original source information. NCMA, on the other hand, is the first realized multiple access scheme that establishes the usefulness of PNC in a non-relay setting, where the AP desires the original source information, yet no self-information or side information is available at the AP.

To exploit PNC and MUD in an integrated manner, in NCMA, at the sender end, each message is partitioned and channel-coded into multiple packets at the MAC layer. These packets are then forwarded to the PHY layer, where another layer of channel coding is performed before the packets are transmitted over the air. At the receiver end, two PHY-layer decoders are used: a PNC decoder attempts to decode the signals simultaneously transmitted by several senders into network-coded packets; at the same time, a conventional MUD decoder attempts to decode the signals into the individual native packets of the two users. The PHY-layer packets collected from different time slots, network-coded as well as native, are then used to decode the MAC-layer messages from the users. We reported an NCMA software-radio prototype in [1], demonstrating that combining the use of PNC decoding and MUD under the framework of NCMA can boost throughput significantly in multiple-access networks.

Our first prototype [1], however, left open a number of issues, the resolution of which is critical to the adoption of NCMA in real practice. The current paper addresses these challenges and makes following contributions: 


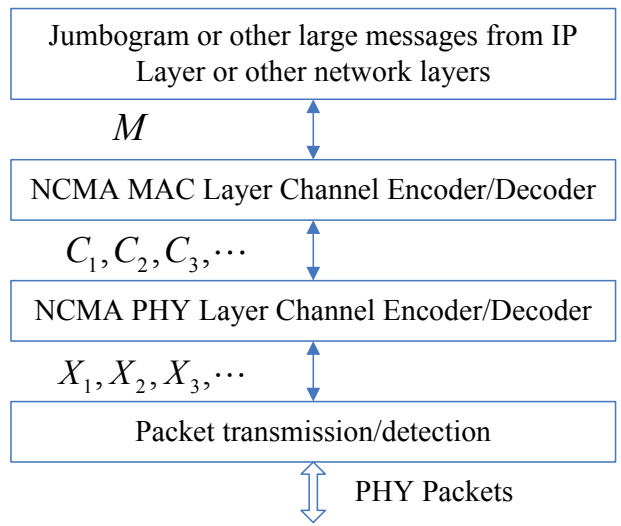

Fig. 2: The architecture of an NCMA node.

1) In [1], we did not demonstrate a system that can operate in real-time. Specifically, although our PHY-layer decoding was done in real-time, our MAC-layer decoding was done offline. The current paper reports an integrated NCMA system with real-time PHY-layer decoding and MAC-layer decoding.

2) In [1], we used three linear equation systems for MAClayer decoding - two for the decoding of the native messages of the two users, and one for the decoding of a network-coded message of the two users. An interesting question is whether there is a framework for a single unified equation system that can yield higher throughput with faster decoding. The current paper provides an affirmative answer. Experimental results demonstrate real-time throughput improvement in excess of $100 \%$ at medium-high SNR $(\geq 8 \mathrm{~dB})$ compared with our previous NCMA prototype [1].

3) In [1], the throughput gain of NCMA was demonstrated for the mid-range SNR (10dB). At low SNR (7.5dB), the traditional time-division multiple-access (TDMA) approach had better performance than NCMA. The current paper presents new decoding techniques that overcome the poor performance at low SNR. Experimental result$\mathrm{s}$ demonstrate performance improvement in excess of $100 \%$ compared with our previous NCMA prototype [1], and performance comparable to the traditional TDMA approach, at low SNR (7.5dB).

\section{NCMA OVERVIEW}

This section first reviews the key concepts of NCMA and then gives a preview of our new MAC-layer decoding framework.

\section{A. NCMA Architecture}

Fig. 2 shows the architecture of an NCMA node ${ }^{1}$. The MAC layer of an NCMA node makes use of an erasure channel code to encode a message $M$ into multiple packets, $C_{1}, C_{2}, \ldots, C_{N}$. In [1] and in this paper, we assume the use

\footnotetext{
${ }^{1}$ For legacy compatibility, an NCMA node can revert to 802.11 operation when necessary (see [1] for details).
}

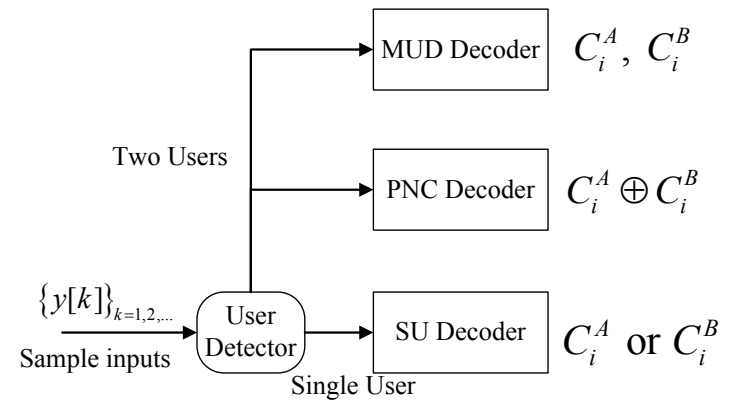

Fig. 3: NCMA PHY-layer channel decoding makes use of single-user (SU) decoder, a MUD decoder and a PNC decoder.

of a Reed Solomon (RS) code at the MAC layer; other erasure channel codes are also possible.

At the PHY layer, each packet $C_{i}$ is further channel-coded into a packet $X_{i}$. In [1] and in this paper, we assume the use of a convolutional code at the PHY layer; other channel codes are also possible. A nice property of the RS code is that as long as the number of PHY-layer packets successfully received and decoded at the AP is above a threshold $L$, then regardless of which $L$ of the $N$ packets $C_{1}, C_{2}, \ldots, C_{N}$ are received correctly, the MAC-layer source message $M$ can be decoded based on the $L$ packets.

In NCMA, each user node transmits packets $X_{1}, X_{2}, \ldots, X_{N}$ to the AP in successive time slots. As illustrated in Fig. 1, two user nodes may transmit in the same time slot. A packet will not be retransmitted even if it cannot be received successfully. Also, the AP will not return acknowledgements for individual packets; it will return an acknowledgement only when the overall message $M$ has been decoded [1].

\section{B. NCMA PHY-layer Decoding and Bridging}

The PHY layer of NCMA makes use of one single-user (SU) decoder and two multiuser decoders, as shown in Fig. 3. The two multiuser decoders are a MUD decoder and a PNC decoder. The SU decoder is used when only one node transmits to the AP. When two nodes A and B transmit to the AP simultaneously, the MUD decoder attempts to decode both $C_{i}^{A}$ and $C_{i}^{B}$ based on the overlapped signals of $X_{i}^{A}$ and $X_{i}^{B}$; the PNC decoder, on the other hand, attempts to decode $C_{i}^{A} \oplus C_{i}^{B}$ (the bit-wise XOR of $C_{i}^{A}$ and $C_{i}^{B}$ ).

Eight Possible Decoding Events - For the MUD decoder, there are four possible outcomes: (i) both $C_{i}^{A}$ and $C_{i}^{B}$ are successfully decoded; (ii) only $C_{i}^{A}$ is successfully decoded; (iii) only $C_{i}^{B}$ is successfully decoded; (iv) neither $C_{i}^{A}$ nor $C_{i}^{B}$ can be decoded. For the PNC decoder, there are two possible outcomes: (a) $C_{i}^{A} \oplus C_{i}^{B}$ is successfully decoded; (b) $C_{i}^{A} \oplus C_{i}^{B}$ cannot be decoded. As a result we have $4 \times 2=8$ possible combined outcomes. We refer to these combined outcomes as "events". Fig. 4 shows a toy example in which the eight possible events occur in successive time slots.

Leveraging Complementary XOR Packets (PHY-layer Bridging) - In Fig. 4, event (ii)(a) and event (iii)(a) occur in 
time slots 3 and 4, in which $C_{3}^{A}$ and $C_{3}^{A} \oplus C_{3}^{B}$ (abbreviated as $C_{3}^{A \oplus B}$ ), and $C_{4}^{B}$ and $C_{4}^{A \oplus B}$, are decoded, respectively. In these two cases, the complementary XOR packets, $C_{3}^{A \oplus B}$ and $C_{4}^{A \oplus B}$, can be used to recover the missing native packets, $C_{3}^{B}$ and $C_{4}^{B}$, respectively - we refer to this process as PHY-layer bridging. With PHY-layer bridging, events (ii)(a) and (iii)(a) are equivalent to event (i) in that both native packets can be obtained.

\section{MAC-layer Decoding and Bridging Based on Three Equa- tion Systems (TES)}

With respect to Fig. 4, event (iv)(a) occurs in time slot 7. None of the native packets can be decoded by the MUD decoder; on the other hand, the PNC decoder can decode $C_{7}^{A} \oplus C_{7}^{B}$. Such lone $C_{i}^{A} \oplus C_{i}^{B}$ are not useful as far as the recovery of the native packets is concerned. Yet, experimental data indicate that event (iv)(a) is not negligible. As shown in Fig. 10 of [1], the event of lone XOR packet, annotated as X in the figure, occurs around $20 \%$ of the time at $\mathrm{SNR}=8.5 \mathrm{~dB}$. This suggests that the system performance could be improved by much if we can find ways to exploit the lone XOR packets.

In the following, we explain with a simple example how MAC-layer channel coding contributes in that regard, assuming the use of the three equation systems (TES) as in [1].

Leveraging Lone XOR Packets (MAC-layer Bridging) Recall from Sec. II-A that a user message $M$ is coded into multiple packets $C_{1}, C_{2}, \ldots, C_{N}$ at the MAC layer using an erasure code. If an RS code is used, then as long as the number of packets received is above a threshold, then regardless of which of the packets are received, the message $M$ can be decoded. For linear codes (note that RS codes are linear), MAC-layer decoding is essentially solving linear equations (to be elaborated in Sec. III). Fig. 5 shows three equation systems (TES) for MAC-layer decoding. In Fig. 5, each column in a table contains the equations of a linear equation system. Specifically, $E q^{A}, E q^{B}, E q^{A \oplus B}$ contain packets of types $C_{i}^{A}$, $C_{i}^{B}, C_{i}^{A \oplus B}$ that can be used to decode $M^{A}, M^{B}, M^{A} \oplus M^{B}$, respectively. The left table in the figure shows the available packets delivered from the PHY layer to the MAC layer. In this example, $L=4$. Thus, from the table, we see that it is possible to decode $M^{A}$ given that there are four packets of type $C_{i}^{A}$. It is not possible to decode $M^{B}$ because there are only three packets of type $C_{i}^{B}$. However, as shown on the right table, being able to decode $M^{A}$ allows us to obtain $C_{i}^{A} \forall i$, not just the four $C_{i}^{A}$ from the PHY layer. In particular, with $C_{2}^{A}$ $\left(C_{6}^{A}\right)$, the previous lone XOR packet $C_{2}^{A \oplus B}\left(C_{6}^{A \oplus B}\right)$ become a complementary XOR packet, allowing $C_{2}^{B}\left(C_{6}^{B}\right)$, shown in red circle in the figure, to be obtained. We refer to this process as MAC-layer bridging. With MAC-layer bridging, $E q^{B}$ now has more than four equations, allowing $M^{B}$ to be decoded.

\section{MAC-layer Decoding and Bridging Based on Unified E- quation System (UES)}

TES in the above does not exploit the PHY packets in a seamless way, since MAC-layer bridging can only be launched when the solution for one of the three equation systems is found. With respect to Fig. 5(a), eight time slots are required to solve for $M^{A}$ and $M^{B}$ in TES. Potentially, there is a chance that we can solve for $M^{A}$ and $M^{B}$ by the end of the seventh time slot. Specifically, by treating $M^{A}$ and $M^{B}$ as a joint message pair, $\left[M^{A} M^{B}\right]^{T}$, we can form a unified equation system (UES) that contain $2 L=8$ equations by the end of the seventh time slot. The precise formulation of UES will be detailed in Sec. III-C. For now, for a rough idea, let us reexamine the left table in Fig. 5. With respect to the message pair $\left[M^{A} M^{B}\right]^{T}$, time slots $1,2,3,5,6$, and 7 each gives us a packet (i.e., an equation), for a total of six equations. Time slot 4 gives us three packets, $C_{4}^{A}, C_{4}^{B}$ and $C_{4}^{A \oplus B}$. However, only two of the packets are independent (e.g., $C_{4}^{A \oplus B}$ can be derived from $C_{4}^{A}$ and $C_{4}^{B}$ ). Thus, time slot 4 gives us two equations. Altogether, time slots 1 to 7 give us eight equations. If these eight equations are linearly independent, we can decode $\left[M^{A} M^{B}\right]^{T}$ in one shot.

\section{UNIFIED MAC-LAYER DECODER}

This section formulates the MAC-layer decoding problem in matrix form. Sec. III-A first presents the formulation for TES [1] to set up the background. Sec. III-B formulates an alternative TES scheme - new in this paper - in which the two user nodes can use different MAC channel codes. Building on this formulation, we present our new single-equation system, UES, in Sec. III-C. Sec. III-D analyzes the performance of UES.

\section{A. Three-Equation System}

TES uses three equation systems for MAC-layer message decoding: $E q^{A}$ for decoding message $M^{A}, E q^{B}$ for decoding message $M^{B}$, and $E q^{A \oplus B}$ for decoding the $\mathrm{XOR}^{2}$ message $M^{A} \oplus M^{B}$.

Suppose that $M^{A}$ and $M^{B}$ each consists of $L K$ bytes arranged in the form of an $L \times K$ matrix, where each byte is an element of the finite field $G F\left(2^{8}\right)$. Assume an $(N, L)$ RS code is used. Furthermore, nodes A and B use the same RS code. Let $G$ be the $N \times L$ generator matrix of the RS code. The $i$ th PHY-layer source packets of nodes A and B, each consisting of $K$ bytes, are generated by

$$
\begin{aligned}
C_{i}^{A} & =G_{i} M^{A}, \\
C_{i}^{B} & =G_{i} M^{B},
\end{aligned}
$$

where $G_{i}$ is the $i$ th row of $G$. In addition, the sequence of packets transmitted by node $\mathrm{A}$ is aligned with the sequence of packets transmitted by node B. In other words, the indexes of the packets simultaneously transmitted by nodes $\mathrm{A}$ and $\mathrm{B}$ are aligned, i.e., they transmit $\left(C_{1}^{A}, C_{1}^{B}\right),\left(C_{2}^{A}, C_{2}^{B}\right), \ldots,\left(C_{N}^{A}, C_{N}^{B}\right)$ in successive time slots.

Consider a particular time slot in which node A transmits $C_{i}^{A}$, and node $\mathrm{B}$ transmits $C_{i}^{B}$. There are four possible outcomes as far as the packets forwarded to the MAC layer is concerned:

\section{Four Possible MAC-Layer Reception Outcomes}

${ }^{2}$ XOR means bit-wise XOR here. It is the simplest implementation of network coding. To simplify explanation, we will assume the bit-wise XOR here. 


\begin{tabular}{|c|c|c|c|}
\hline Time Slot & $A$ & $A \oplus B$ & $B$ \\
\hline 1 & $C_{1}^{A}$ & $C_{1}^{A \oplus B}$ & $C_{1}^{B}$ \\
\hline 2 & $C_{2}^{A}$ & $\varnothing$ & $C_{2}^{B}$ \\
\hline 3 & $C_{3}^{A}$ & $C_{3}^{A \oplus B}$ & $\varnothing$ \\
\hline 4 & $\varnothing$ & $C_{4}^{A \oplus B}$ & $C_{4}^{B}$ \\
\hline 5 & $C_{5}^{A}$ & $\varnothing$ & $\varnothing$ \\
\hline 6 & $\varnothing$ & $\varnothing$ & $C_{6}^{B}$ \\
\hline 7 & $\varnothing$ & $C_{7}^{A \oplus B}$ & $\varnothing$ \\
\hline 8 & $\varnothing$ & $\varnothing$ & $\varnothing$ \\
\hline
\end{tabular}

Fig. 4: An example of PHY-layer packet reception patterns for concurrently transmitted packets. Empty entries $(\phi)$ mean the corresponding packets cannot be decoded. Each column is labeled $J$ (e.g., $A \oplus B$ ) corresponding to packet type $J$ (e.g., $A \oplus B)$ being decoded.
Example: Decoding $M^{B}$, based on $M^{A}$ and $M^{A} \oplus M^{B}$, with $L=4$

\begin{tabular}{|c|c|c|c|}
\hline $\begin{array}{c}\text { Time } \\
\text { Slot }\end{array}$ & $E q^{A}$ & $E q^{A \oplus B}$ & $E q^{B}$ \\
\hline 1 & $C_{1}^{A}$ & & \\
\hline 2 & & $C_{2}^{A \oplus B}$ & \\
\hline 3 & & & $C_{3}^{B}$ \\
\hline 4 & $C_{4}^{A}$ & $C_{4}^{A \oplus B}$ & $C_{4}^{B}$ \\
\hline 5 & $C_{5}^{A}$ & & \\
\hline 6 & & $C_{6}^{A \oplus B}$ & \\
\hline 7 & & & $C_{7}^{B}$ \\
\hline 8 & $C_{8}^{A}$ & & \\
\hline
\end{tabular}

(a)

\begin{tabular}{|c|c|c|c|c|}
\hline \multirow{9}{*}{$\begin{array}{c}\text { Sequential } \\
\text { Decoding } \\
\text { Algorithm in } \\
\stackrel{\text { TES }}{\longrightarrow}\end{array}$} & $\begin{array}{l}\text { Time } \\
\text { Slot }\end{array}$ & $E q^{A}$ & $E q^{A \oplus B}$ & $E q^{B}$ \\
\hline & 1 & $C_{1}^{A}$ & & \\
\hline & 2 & $\left(C_{2}^{A}\right)$ & $C_{2}^{A \oplus B}$ & $\left(C_{2}^{B}\right)$ \\
\hline & 3 & $\left(\overline{\left.C_{3}^{A}\right)}\right.$ & & $C_{3}^{B}$ \\
\hline & 4 & $C_{4}^{A}$ & $C_{4}^{A \oplus B}$ & $C_{4}^{B}$ \\
\hline & 5 & $C_{5}^{A}$ & & \\
\hline & 6 & $\left(C_{6}^{A}\right)$ & $C_{6}^{A \oplus B}$ & $\left(C_{6}^{B}\right)$ \\
\hline & 7 & $\left(C_{7}^{A}\right)$ & & $C_{7}^{B}$ \\
\hline & 8 & $C_{8}^{A}$ & & \\
\hline
\end{tabular}

(b)
Fig. 5: NCMA decoding process, with $L=4$ in the RS code as an example.

1) The MAC layer receives $C_{i}^{A}, C_{i}^{B}$, and $C_{i}^{A} \oplus C_{i}^{B}$ - As long as at least two of $C_{i}^{A}, C_{i}^{B}$, or $C_{i}^{A} \oplus C_{i}^{B}$ are decoded by the MUD and PNC decoders, by PHY-layer bridging, all three packets can be delivered to the MAC layer.

2) The MAC layer receives only $C_{i}^{A}$ or $C_{i}^{B}$ - This is the case if MUD decoding yields either $C_{i}^{A}$ or $C_{i}^{B}$, but PNC decoding is not successful.

3) The MAC layer receives only XOR packet $C_{i}^{A} \oplus C_{i}^{B}$ - This is the case if PNC decoding is successful, but MUD decoding yields no packet.

4) The MAC layer receives no packet - This is the case if both the MUD and PNC decoders fail to decode any packet.

If a native packet, $C_{i}^{A}$ or $C_{i}^{B}$, is received, a new equation in the form of (1) is made available for MAC-layer decoding. If an XOR packet $C_{i}^{A} \oplus C_{i}^{B}$ is received, a new equation in the following form is made available instead:

$$
C_{i}^{A} \oplus C_{i}^{B}=G_{i} M^{A} \oplus G_{i} M^{B}=G_{i}\left(M^{A} \oplus M^{B}\right) .
$$

Over time, the MAC layer of the AP obtains vectors of packets from the PHY layer. Let $\tilde{C}^{A}\left(\tilde{C}^{B}\right)$ be a vector containing the native packets $C_{i}^{A}\left(C_{i}^{B}\right)$ it receives, and $\tilde{C}^{A \oplus B}$ be a vector containing the XOR packets $C_{i}^{A} \oplus C_{i}^{B}$ it receives. Gathering together the received packets, we have three equation systems, referred to as $E q^{A}, E q^{B}$, and $E q^{A \oplus B}$, respectively, as follows:

$$
\begin{aligned}
& \tilde{G}^{A} M^{A}=\tilde{C}^{A}, \\
& \tilde{G}^{B} M^{B}=\tilde{C}^{B}, \\
& \tilde{G}^{X}\left(M^{A} \oplus M^{B}\right)=\tilde{C}^{A \oplus B},
\end{aligned}
$$

where $\tilde{G}^{A}, \tilde{G}^{B}$, and $\tilde{G}^{X}$ consist of the subsets of the rows of $G$ corresponding to the received $\tilde{C}^{A}, \tilde{C}^{B}$, and $\tilde{C}^{A \oplus B}$, respectively. In general, some rows in $\tilde{G}^{A}, \tilde{G}^{B}$, and $\tilde{G}^{X}$ are common rows drawn from the same rows in $G$, while other rows are not, depending on whether we have outcome 1), 2), 3 ), or 4) in the above.

As the MAC layer receives more and more packets, the numbers of rows in $\tilde{G}^{A}, \tilde{G}^{B}$, and $\tilde{G}^{X}$ in (3) increase. Suppose that at one point in time, we have $L$ rows in $\tilde{G}^{A}$, but fewer than $L$ rows in $\tilde{G}^{B}$ and $\tilde{G}^{X}$. Then, thanks to the property of RS codes, $\tilde{G}^{A}$ is full rank and we can decode $M^{A}$. Through MAClayer bridging, however, we can obtain additional equations for $E q^{B}$ through $E q^{A \oplus B}$, as follows.

First, we retain only those rows in $\tilde{G}^{X}$ that correspond to lone XOR packets, i.e., we retain the XOR equations obtained through outcome 3 ) but remove the XOR equations obtained through outcome 1). Let $\bar{G}^{X}\left(M^{A} \oplus M^{B}\right)=\bar{C}^{A \oplus B}$ be the resulting $E q^{A \oplus B}$ that consisting of the lone XOR packets only. Given that $M^{A}$ is decoded, $E q^{A \oplus B}$ can be rewritten as $\bar{G}^{X} M^{B}=\bar{C}^{A \oplus B} \oplus \bar{G}^{X} M^{A}=\bar{C}^{A \oplus B} \oplus \bar{G}^{X}\left(\tilde{G}^{A}\right)^{-1} \tilde{C}^{A}$ . Combining this with $\tilde{G}^{B} M^{B}=\tilde{C}^{B}$ in (3), we have a new $E q^{B}$ :

$$
\left[\begin{array}{l}
\bar{G}^{X} \\
\tilde{G}^{B}
\end{array}\right] M^{B}=\left[\begin{array}{c}
\bar{C}^{A \oplus B} \oplus \bar{G}^{X}\left(\tilde{G}^{A}\right)^{-1} \tilde{C}^{A} \\
\tilde{C}^{B}
\end{array}\right] .
$$

If $\left[\begin{array}{c}\bar{G}^{X} \\ \tilde{G}^{B}\end{array}\right]$ now has $L$ rows, then $\left[\begin{array}{c}\bar{G}^{X} \\ \tilde{G}^{B}\end{array}\right]$ is full rank and $M^{B}$ can also be decoded. If not, we need additional equations for $E q^{B}$ to solve for $M^{B}$. This means node B must transmit more packets generated from $M^{B}$. Node B can pair $M^{B}$ with a new message from node A or a message from another node (say node C) for its subsequent packets. The XOR equations in (3) will not be useful anymore, but the $E q^{B}$ equations in (4) remain useful and not wasted when $M^{B}$ is paired with another message.

Similarly, if we have $L$ rows in $\tilde{G}^{B}$, but fewer than $L$ rows in $\tilde{G}^{A}$ and $\tilde{G}^{X}$, the new $E q^{A}$ after MAC-layer bridging is

$$
\left[\begin{array}{l}
\bar{G}^{X} \\
\tilde{G}^{A}
\end{array}\right] M^{A}=\left[\begin{array}{c}
\bar{C}^{A \oplus B} \oplus \bar{G}^{X}\left(\tilde{G}^{B}\right)^{-1} \tilde{C}^{B} \\
\tilde{C}^{A}
\end{array}\right] .
$$

Yet another possibility is that we have $L$ rows in $G^{X}$ while there are fewer than $L$ rows in $\tilde{G}^{A}$ and $\tilde{G}^{B}$. This allows $M^{A} \oplus$ $M^{B}$ to be decoded, i.e., $M^{A} \oplus M^{B}=\left(\tilde{G}^{X}\right)^{-1} \tilde{C}^{A \oplus B}$.

Meanwhile, $E q^{B}$ is $\tilde{G}^{B} M^{B}=\tilde{C}^{B}$. First, we retain only those rows in $\tilde{G}^{B}$ that are not the same as any row in $\tilde{G}^{A}$, i.e., we retain the equations in $\tilde{G}^{B} M^{B}=\tilde{C}^{B}$ obtained through outcome 2) but remove those equations obtained through outcome 1). Let $\bar{G}^{B} M^{B}=\bar{C}^{B}$ be the resulting equations. Through MAC-layer bridging, we can get additional equations for $E q^{A}$ in the form of $\bar{G}^{B} M^{A}=\bar{C}^{B} \oplus \bar{G}^{B}\left(\tilde{G}^{X}\right)^{-1} \tilde{C}^{A \oplus B}$. 
By the same token, we can get additional equations for $E q^{B}$ in the form of $\bar{G}^{A} M^{B}=\bar{C}^{A} \oplus \bar{G}^{A}\left(\tilde{G}^{X}\right)^{-1} \tilde{C}^{A \oplus B}$. Thus, the new $E q^{A}$ and $E q^{B}$ are respectively

$$
\begin{aligned}
& {\left[\begin{array}{l}
\bar{G}^{B} \\
\tilde{G}^{A}
\end{array}\right] M^{A}=\left[\begin{array}{c}
\bar{C}^{B} \oplus \bar{G}^{B}\left(\tilde{G}^{X}\right)^{-1} \tilde{C}^{A \oplus B} \\
\tilde{C}^{A}
\end{array}\right],} \\
& {\left[\begin{array}{l}
\bar{G}^{A} \\
\tilde{G}^{B}
\end{array}\right] M^{B}=\left[\begin{array}{c}
\bar{C}^{A} \oplus \bar{G}^{A}\left(\tilde{G}^{X}\right)^{-1} \tilde{C}^{A \oplus B} \\
\tilde{C}^{B}
\end{array}\right] .}
\end{aligned}
$$

If $\left[\begin{array}{c}\bar{G}^{B} \\ \tilde{G}^{A}\end{array}\right]$ or $\left[\begin{array}{c}\bar{G}^{A} \\ \tilde{G}^{B}\end{array}\right]$ is full rank, we can decode $M^{A}$ or $M^{B}$, respectively. If neither $M^{A}$ or $M^{B}$ is decoded at this time, then more packets generated from $M^{A}$ and $M^{B}$ will be transmitted by node $\mathrm{A}$ and node $\mathrm{B}$. Note that for these subsequent packets, outcome 2) is equivalent to outcome 1), since $M^{A} \oplus M^{B}$ has been solved. For example, if the MAC layer receives only $C_{i}^{A}$ under outcome 2), then $C_{i}^{B}$ can also be obtained by $C_{i}^{B}=$ $C_{i}^{A} \oplus G_{i}^{A}\left(\tilde{G}^{X}\right)^{-1} \tilde{C}^{A \oplus B}$.

In the above, we have assumed that the parameter $L$ is the same for both nodes $\mathrm{A}$ and $\mathrm{B}$. In general, nodes $\mathrm{A}$ and $\mathrm{B}$ can use different $L$ (i.e., $L_{A} \neq L_{B}$ ). The interested reader is referred to [1] for the treatment of TES where $L_{A} \neq L_{B}$.

\section{B. Dissimilar MAC-layer Codes}

So far, we have assumed that nodes $\mathrm{A}$ and $\mathrm{B}$ use the same MAC-layer channel code, and the MAC protocol must coordinate nodes A and B to ensure the packets simultaneously transmitted by them are aligned in index. This complicates the MAC protocol operation. We now remove this limitation and propose a modified TES that allows nodes $\mathrm{A}$ and $\mathrm{B}$ to use different channel codes and that does not require packet alignment.

Assuming $(N, L)$ RS codes, instead of a common matrix $G$, let $G_{A}$ and $G_{B}$ be the generator matrices of nodes $\mathrm{A}$ and B, respectively $-G_{A}$ and $G_{B}$ can be generated by permuting rows of $G$ randomly. Then the three-equation system can be rewritten as

$$
\begin{aligned}
& \tilde{G}_{A}^{A} M^{A}=\tilde{C}^{A}, \\
& \tilde{G}_{B}^{B} M^{B}=\tilde{C}^{B}, \\
& \tilde{G}_{A}^{X} M^{A} \oplus \tilde{G}_{B}^{X} M^{B}=\tilde{C}^{A \oplus B},
\end{aligned}
$$

where $\tilde{G}_{A}^{A}, \tilde{G}_{B}^{B}, \tilde{G}_{A}^{X}$ and $\tilde{G}_{B}^{X}$ consist of subsets of the rows of $G_{A}$ and $G_{B}$ corresponding to the packets in $\tilde{C}^{A}, \tilde{C}^{B}$, and $\tilde{C}^{A \oplus B}$, respectively.

The MAC-layer bridging mechanism can also be rewritten. For the case where $M^{A}$ is decoded first, the new $E q^{B}$ is

$$
\left[\begin{array}{c}
\bar{G}_{B}^{X} \\
\tilde{G}_{B}^{B}
\end{array}\right] M^{B}=\left[\begin{array}{c}
\bar{C}^{A \oplus B} \oplus \bar{G}_{A}^{X}\left(\tilde{G}_{A}^{A}\right)^{-1} \tilde{C}^{A} \\
\tilde{C}^{B}
\end{array}\right] .
$$

For the case where $M^{B}$ is decoded first, the new $E q^{A}$ is

$$
\left[\begin{array}{c}
\bar{G}_{A}^{X} \\
\tilde{G}_{A}^{A}
\end{array}\right] M^{A}=\left[\begin{array}{c}
\bar{C}^{A \oplus B} \oplus \bar{G}_{B}^{X}\left(\tilde{G}_{B}^{B}\right)^{-1} \tilde{C}^{B} \\
\tilde{C}^{A}
\end{array}\right] .
$$

For the case where $E q^{A \oplus B}$ is solved first $\left(\tilde{G}_{A}^{X}\right.$ and $\tilde{G}_{B}^{X}$ are full rank) ${ }^{3}$, we can express $E q^{A \oplus B}$ as $\left(\tilde{G}_{B}^{X}\right)^{-1} \tilde{G}_{A}^{X} M^{A} \oplus M^{B}=$ $\left(\tilde{G}_{B}^{X}\right)^{-1} \tilde{C}^{A \oplus B}$ and $M^{A} \oplus\left(\tilde{G}_{A}^{X}\right)^{-1} \tilde{G}_{B}^{X} M^{B}=\left(\tilde{G}_{A}^{X}\right)^{-1} \tilde{C}^{A \oplus B}$. The new $E q^{A}$ and $E q^{B}$ are respectively

$$
\begin{aligned}
& {\left[\begin{array}{c}
\bar{G}_{B}^{B}\left(\tilde{G}_{B}^{X}\right)^{-1} \tilde{G}_{A}^{X} \\
\tilde{G}_{A}^{A}
\end{array}\right] M^{A}=\left[\begin{array}{c}
\bar{C}^{B} \oplus \bar{G}_{B}^{B}\left(\tilde{G}_{B}^{X}\right)^{-1} \tilde{C}^{A \oplus B} \\
\tilde{C}^{A}
\end{array}\right],} \\
& {\left[\begin{array}{c}
\bar{G}_{A}^{A}\left(\tilde{G}_{A}^{X}\right)^{-1} \tilde{G}_{B}^{X} \\
\tilde{G}_{B}^{B}
\end{array}\right] M^{B}=\left[\begin{array}{c}
\bar{C}^{A} \oplus \bar{G}_{A}^{A}\left(\tilde{G}_{A}^{X}\right)^{-1} \tilde{C}^{A \oplus B} \\
\tilde{C}^{B}
\end{array}\right] .}
\end{aligned}
$$

In addition, if $E q^{A \oplus B}$ is solved first, for the subsequent packets, outcome 2) is equivalent to outcome 1) in terms of getting new equations. For example, if the MAC layer receives only $C_{i}^{A}$ under outcome 2), then the MAC layer can obtain a new equation for $M^{B}: G_{i}^{A}\left(\tilde{G}_{A}^{X}\right)^{-1} \tilde{G}_{B}^{X} M^{B}=$ $C_{i}^{A} \oplus G_{i}^{A}\left(\tilde{G}_{A}^{X}\right)^{-1} \tilde{G}_{B}^{X} \tilde{C}^{A \oplus B}$.

\section{Unified Equation System}

We now present a unified equation system for message decoding. Our new unified equation system, UES, treats $\left[\begin{array}{ll}M_{A} & M_{B}\end{array}\right]^{T}$ as a joint message, and constructs equations that model all reception outcomes in the MAC layer. The unified equation system is of the following form:

$$
\tilde{G}\left[\begin{array}{c}
M^{A} \\
M^{B}
\end{array}\right] \triangleq\left[\begin{array}{cc}
\tilde{G}_{A}^{A} & 0 \\
\bar{G}_{A}^{X} & \bar{G}_{B}^{X} \\
0 & \tilde{G}_{B}^{B}
\end{array}\right]\left[\begin{array}{c}
M^{A} \\
M^{B}
\end{array}\right]=\left[\begin{array}{c}
\tilde{C}^{A} \\
\bar{C}^{A \oplus B} \\
\tilde{C}^{B}
\end{array}\right] .
$$

UES (11) is constructed as follows. Recall that $G_{A}$ and $G_{B}$ are the generator matrices at source nodes $\mathrm{A}$ and $\mathrm{B}$. Let the $i$ th row of $G_{A}$ be $G_{A, i}$, and the $i$ th row of $G_{B}$ be $G_{B, i}$. For MAC-layer reception outcome 1$), C_{i}^{A}, C_{i}^{B}$ and $C_{i}^{A} \oplus C_{i}^{B}$ are received. Instead of using all three equations as in TES we only add two equations $\left[\begin{array}{cc}G_{A, i} & 0 \\ 0 & G_{B, i}\end{array}\right]\left[\begin{array}{l}M^{A} \\ M^{B}\end{array}\right]=\left[\begin{array}{l}C_{i}^{A} \\ C_{i}^{B}\end{array}\right]$ to (11); the XOR equation for $C_{i}^{A} \oplus C_{i}^{B}$ will not be used because the information is already contained in two equations of $C_{i}^{A}$ and $C_{i}^{B}$ and it would be a redundant equation within (11) if it were added. The action for outcome 2) is similar to that in TES: if the PHY-layer can only provide $C_{i}^{A}$ (or $C_{i}^{B}$ ), then we add one equation $\left[\begin{array}{ll}G_{A, i} & 0\end{array}\right]\left[\begin{array}{l}M^{A} \\ M^{B}\end{array}\right]=C_{i}^{A}$ (or $\left[\begin{array}{ll}0 & G_{B, i}\end{array}\right]\left[\begin{array}{l}M^{A} \\ M^{B}\end{array}\right]=$ $C_{i}^{B}$ ) to (6). Finally, for outcome 3) the PHY-layer can only provide a lone XOR packet $C_{i}^{A \oplus B}=C_{i}^{A} \oplus C_{i}^{B}$ : we add one equation $\left[\begin{array}{ll}G_{A, i} & G_{B, i}\end{array}\right]\left[\begin{array}{l}M^{A} \\ M^{B}\end{array}\right]^{i}=C_{i}^{A \oplus B}$ to (11).

Over time, the MAC layer accumulates packets from the PHY layer. In (11), $\tilde{C}^{A}\left(\tilde{C}^{B}\right)$ is a vector consisting of the set of native packets $C_{i}^{A}\left(C_{i}^{B}\right)$ the MAC layer receives, $\bar{C}^{A \oplus B}$ is a vector consisting of the set of lone XOR packets $C_{i}^{A} \oplus C_{i}^{B}$ it receives, and $\tilde{G}_{A}^{A}, \tilde{G}_{B}^{B}, \tilde{G}_{A}^{X}$, and $\tilde{G}_{B}^{X}$ consist of the subsets of the rows of $G_{A}$ and $G_{B}$ corresponding to the packets in $\tilde{C}^{A}, \tilde{C}^{B}$, and $\bar{C}^{A \oplus B}$, respectively.

At some point in time, if the coupled matrix $\tilde{G}=$ $\left[\begin{array}{cc}\tilde{G}_{A}^{A} & 0 \\ \bar{G}_{A}^{X} & \bar{G}_{B}^{X} \\ 0 & \tilde{G}_{B}^{B}\end{array}\right]$ is full rank, then we can solve for both $M^{A}$

${ }^{3}$ In this case, by saying $E q^{A \oplus B}$ is solved, we do not mean the bitwise XOR message $M^{A} \oplus M^{B}$ can be decoded. Rather, we mean the network-coded messages in the form of $\left(\tilde{G}_{B}^{X}\right)^{-1} \tilde{G}_{A}^{X} M^{A} \oplus M^{B}$ and $M^{A} \oplus\left(\tilde{G}_{A}^{X}\right)^{-1} \tilde{G}_{B}^{X} M^{B}$ can be decoded. 
and $M^{B}$ in one shot. However, if $\tilde{G}_{A}^{A}$ has more than $L$ rows before $\tilde{G}$ is full rank, we can decode $M^{A}$ first and then obtain more equations related $M^{B}$ through MAC-layer bridging as in (8) and (9).

Summarizing, there are two conditions under which $\tilde{G}$ yields successful message decoding:

Condition 1: $\tilde{G}_{A}^{A}$ has fewer than $L$ rows, $\tilde{G}_{B}^{B}$ has fewer than $L$ rows, but $\tilde{G}$ is full rank.

Condition 2: $\tilde{G}_{A}^{A}$ has $L$ rows (or $\tilde{G}_{B}^{B}$ has $L$ rows), and $\tilde{G}$ is not full rank.

Compared with TES, condition 1 is new. It is possible that condition 1 is fulfilled in UES (allowing both $M^{A}$ and $M^{B}$ to be decoded), and yet TES cannot decode any message yet (see example in Fig. 5(b)). The next question is whether there are situations in which TES can decode messages, but UES cannot. As argued in the next few paragraphs, the answer is no. Thus, UES is no worse than and may be better than TES.

Consider TES. Let $\tilde{C}^{A \oplus B}$ be a vector consisting of the XOR packets $C_{i}^{A} \oplus C_{i}^{B}$ the MAC layer receives, and $\tilde{G}_{A}^{X}$ and $\tilde{G}_{B}^{X}$ consist of the subsets of the rows of $G_{A}$ and $G_{B}$ corresponding to the packets in $\tilde{C}^{A \oplus B}$. Consider the following matrix equation:

$$
\check{G}\left[\begin{array}{c}
M^{A} \\
M^{B}
\end{array}\right] \triangleq\left[\begin{array}{cc}
\tilde{G}_{A}^{A} & 0 \\
\tilde{G}_{A}^{X} & \tilde{G}_{B}^{X} \\
0 & \tilde{G}_{B}^{B}
\end{array}\right]\left[\begin{array}{c}
M^{A} \\
M^{B}
\end{array}\right]=\left[\begin{array}{c}
\tilde{C}^{A} \\
\tilde{C}^{A \oplus B} \\
\tilde{C}^{B}
\end{array}\right] .
$$

We note that $E q^{A}, E q^{B}$, and $E q^{A \oplus B}$ in TES is fully embedded in (12): each equation appearing in $E q^{A}, E q^{B}$, or $E q^{A \oplus B}$ also appears in (12). Furthermore, $\check{G} \triangleq\left[\begin{array}{cc}\tilde{G}_{A}^{A} & 0 \\ \tilde{G}_{A}^{X} & \tilde{G}_{B}^{X} \\ 0 & \tilde{G}_{B}^{B}\end{array}\right]$ is more powerful than TES since it can also be used to decode the joint message if $\check{G}$ is full rank. Thus, a unified system based on $\breve{G}$ will yield performance no worse than that the original TES.

Next, let us compare the two unified equation systems of (11) and (12). Comparing $\tilde{G}$ and $\check{G}$, we find that $\tilde{G}$ is essentially equivalent to $\breve{G}$ in term of message decoding, because $\tilde{G}$ can be obtained by elementary row operations on $\check{G}$. Specifically, by elementary row operations on $\check{G}$, we can obtain $\left[\begin{array}{c}\tilde{G} \\ 0\end{array}\right]$; the redundant equations in $\check{G}$ that are not linearly independent of the equations in $\tilde{G}$ can be removed. Thus, the performances of the unified equation systems under (11) and (12) are the same.

\section{Performance of UES}

This section analyzes when condition 1 for UES (11) as stated in Sec. III-C is satisfied. We re-emphasize that condition 1 captures the situation under which UES may be superior to the TES; under condition 2, the two systems behave the same way. Here, we consider a more general condition that allows $L_{A} \neq L_{B}$ in the equation system. As shown in [1], by truncating columns of the generator matrices $G_{A}$ or $G_{B}$, the generalization is possible. An interesting question is:

Question: Is $\tilde{G}$, the coupled matrix of nodes $\mathrm{A}$ and $\mathrm{B}$, full rank given that $\tilde{G}$ has $L_{A}+L_{B}$ rows?
Note that $L_{A}+L_{B}$ is the minimum number of equations needed to solve for both $M^{A}$ and $M^{B}$. In other words, can we always decode $M^{A}$ and $M^{B}$ with the minimum number of needed equations? In general, the answer is no; however, the decoding probability is close to one.

It is non-trivial to derive the probability of $\tilde{G}$ being full rank when the RS code is used. The probability, however, can be derived exactly if a random code is used. We derive the probability under a random code here; we will present simulation results that show the difference between using random codes and RS codes is small.

For a matrix $Z$, let $\operatorname{rank}(Z), \operatorname{row}(Z)$, and $\operatorname{col}(Z)$ denote its rank, number of rows, and number of columns, respectively. We first give a known result in a lemma:

Lemma 1. [7] Let $Z=\left(z_{i j}\right)$ be an $(r \times n)$-matrix, where $z_{i j} \in G F(t)$ and $r \leq n$. Suppose that $z_{i j}$ are independently and identically distributed (i.i.d.) with uniform distribution: $\operatorname{Pr}\left(z_{i j}=s\right)=1 / t \forall s \in G F(t)$. Then $\operatorname{Pr}(\operatorname{rank}(Z)=r)=$ $\left.\prod_{l=n+1-r}^{n}\left(1-1 / t^{l}\right)\right)$.

The following theorem can be derived based on Lemma 1 .

Theorem 2. Let $Z=\left[\begin{array}{cc}Z^{A} & 0 \\ Z_{A}^{X} & Z_{B}^{X} \\ 0 & Z^{B}\end{array}\right]=\left(z_{i j}\right)$ be an $(r \times$ $\left.\left(n_{A}+n_{B}\right)\right)$ matrix, where $z_{i j} \in G \vec{F}(t)$ and $r \leq n_{A}+n_{B}$; $r_{A}=\operatorname{row}\left(Z^{A}\right)<n_{A}=\operatorname{col}\left(Z^{A}\right) ; r_{B}=\operatorname{row}\left(Z^{B}\right)<n_{B}=$ $\operatorname{col}\left(Z^{B}\right)$. Suppose that $z_{i j}$ are i.i.d. with uniform distribution: $\operatorname{Pr}\left(z_{i j}=s\right)=1 / t \forall s \in G F(t)$. Then $\operatorname{Pr}(\operatorname{rank}(Z)=r)=$ $\prod_{l=n_{A}+1-r_{A}}^{n_{A}}\left(1-1 / t^{l}\right) \prod_{l=n_{B}+1-r_{B}}^{n_{B}}\left(1-1 / t^{l}\right)$ $\prod_{l=n_{A}+n_{B}+1-r_{A}-r_{B}-r_{X}}^{n_{A}+n_{B}-r_{A}-r_{B}}\left(1-1 / t^{l}\right)$.

Proof. Let $E Z$ be the event that $\operatorname{rank}(Z)=r, E A$ be the event that $\operatorname{rank}\left(Z^{A}\right)=r_{A}$, and $E B$ be the event that $\operatorname{rank}\left(Z^{B}\right)=r_{B}$. We have $\operatorname{Pr}(E Z)=\operatorname{Pr}(E Z, E A, E B)=$ $\operatorname{Pr}(E A) \operatorname{Pr}(E B \mid E A) \operatorname{Pr}(E Z \mid E A, E B)$. Since the first $n_{A}$ columns of $\left[0 Z^{B}\right]$ are all zeros, $\operatorname{Pr}(E B \mid E A)=\operatorname{Pr}(E B)$. Hence

$$
\operatorname{Pr}(E Z)=\operatorname{Pr}(E A) \operatorname{Pr}(E B) \operatorname{Pr}(E Z \mid E A, E B) .
$$

According to Lemma 1,

$$
\begin{aligned}
& \operatorname{Pr}(E A)=\prod_{l=n_{A}+1-r_{A}}^{n_{A}}\left(1-1 / t^{l}\right), \\
& \operatorname{Pr}(E B)=\prod_{l=n_{B}+1-r_{B}}^{n_{B}}\left(1-1 / t^{l}\right) .
\end{aligned}
$$

Given that $Z^{A}$ and $Z^{B}$ are both full rank, $Z$ is full rank if and only if that each row in $\left[\begin{array}{ll}Z_{A}^{X} & \left.Z_{B}^{X}\right]\end{array}\right]$ is linearly independent of other rows. The rows in $\left[\begin{array}{cc}Z^{A} & 0 \\ 0 & Z^{B}\end{array}\right]$ span a row-vector space of dimension $r^{A}+r^{B}$ and size $t^{r_{A}+r_{B}}$. Thus, the probability that the first row of $\left[\begin{array}{cc}Z_{A}^{X} & Z_{B}^{X}\end{array}\right]$ is linearly independent of the rows in $\left[\begin{array}{cc}Z^{A} & 0 \\ 0 & Z^{B}\end{array}\right]$ is given by $\left(1-t^{r_{A}+r_{B}} / t^{n_{A}+n_{B}}\right)$. Similarly, given that the first row $\left[\begin{array}{ll}Z_{A}^{X} & Z_{B}^{X}\end{array}\right]$ and the rows in $\left[\begin{array}{cc}Z^{A} & 0 \\ 0 & Z^{B}\end{array}\right]$ are linearly independent, the probability that the next row of $\left[\begin{array}{ll}Z_{A}^{X} & Z_{B}^{X}\end{array}\right]$ is linearly independent of the first row of $\left[\begin{array}{cc}Z_{A}^{X} & Z_{B}^{X}\end{array}\right]$ and the rows in $\left[\begin{array}{cc}Z^{A} & 0 \\ 0 & Z^{B}\end{array}\right]$ is given by 
TABLE I: The probability of matrix $\tilde{G}$ being full rank when using RS codes and random codes. For practical implementations, we are interested in $G F\left(2^{8}\right)$ for the elements in $\tilde{G}$, which corresponds to the case of $N=2^{8}-1=255$ in the above. For other $N$, the results are similar.

\begin{tabular}{|c|c|c|c|c|}
\hline$\left[N, L_{A}, L_{B}\right]$ & {$\left[l_{A}, l_{B}, l_{X}\right]$} & $\begin{array}{c}\text { RS Codes } \\
(\text { Simulation })\end{array}$ & $\begin{array}{c}\text { Random Codes } \\
(\text { Simulation })\end{array}$ & $\begin{array}{c}\text { Random Codes } \\
\text { (Analysis) }\end{array}$ \\
\hline$[255,10,10]$ & {$[5,5,10]$} & 0.996129 & 0.996066 & 0.996078 \\
\hline$[255,10,10]$ & {$[1,9,10]$} & 0.996112 & 0.996086 & 0.996063 \\
\hline$[255,10,10]$ & {$[5,9,6]$} & 0.995970 & 0.996017 & 0.996063 \\
\hline$[255,15,10]$ & {$[1,9,15]$} & 0.996047 & 0.996131 & 0.996063 \\
\hline$[255,15,10]$ & {$[5,9,11]$} & 0.996152 & 0.996162 & 0.996063 \\
\hline
\end{tabular}

$\left(1-t^{r_{A}+r_{B}+1} / t^{n_{A}+n_{B}}\right) ;$ and so on. Therefore,

$$
\operatorname{Pr}(E Z \mid E A, E B)=\prod_{l=n_{A}+n_{B}+1-r_{A}-r_{B}-r_{X}}^{n_{A}+n_{B}-r_{A}-r_{B}}\left(1-1 / t^{l}\right) .
$$
(13).

The proof is completed by substituting (14), and (15) into

We now apply Theorem 2 to find the probability that $\tilde{G}$ is full rank. Let $\operatorname{row}\left(\tilde{G}_{A}^{A}\right)=l_{A}<L_{A}, \operatorname{row}\left(\tilde{G}_{B}^{B}\right)=l_{B}<L_{B}$, and row $\left(\left[\bar{G}_{A}^{X} \bar{G}_{B}^{X}\right]\right)=l_{x}$. Suppose that $\tilde{G}$ has $L_{A}+L_{B}$ rows (i.e., $l_{A}+l_{B}+l_{X}=L_{A}+L_{B}$ ). According to Theorem 2, for $G F\left(2^{8}\right)$ and $L_{A}=L_{B}=10$, the probability of resolving both $M^{A}$ and $M^{B}$ with $L_{A}+L_{B}$ rows is at least 0.996048 (we tried different combination of $\left.\left[l_{A}, l_{B}, l_{X}\right]\right)$. This result shows that the unified equation system is highly efficient in terms of exploiting the equations offered by the PHY layer because it requires the bare minimum number of equations to resolve $M^{A}$ and $M^{B}$ with high probability.

We performed large-scale simulations to verify our analysis. Table I contains the results of different combinations of $\left[l_{A}, l_{B}, l_{X}\right]$ with $l_{A}+l_{B}+l_{X}=L_{A}+L_{B}$. For each $\left[l_{A}, l_{B}, l_{X}\right]$, we generated 100,000 matrices, and count the ratio of matrices that are full rank. We repeated this ten times and present the average results.

From Table I, we see that our analysis of random codes is very close to the simulation results. In addition, the performance of RS codes can be well approximated by the analysis of random codes. The positive results imply that we may replace RS codes with random codes in NCMA. An advantage of random codes is that random codes allow more equations to be generated at the source. For RS codes, the number of equations is limited by size of the finite field we use; for $G F\left(2^{8}\right)$, the RS codes gives $2^{8}-1=255$ equations [1]. A source may run out of equations to send if the AP still cannot decode the message after the source has sent the 255 equations. For random codes, we will almost surely never run out of equations.

For random codes, we should also consider the probability that $G_{A}^{A}$ is full rank if it has $L_{A}$ rows (i.e., when condition 2 is satisfied, $G_{A}^{A}$ may not be full rank under random codes). By Lemma 1 , it is easy to verify that for $G F\left(2^{8}\right)$ and $L_{A}=10$, $G_{A}^{A}$ is full rank with probability $\prod_{l=1}^{L_{A}}\left(1-1 / t^{l}\right) \approx 0.996078$ when $t=2^{8}$. Thus, the number of packets of type $C_{i}^{A}$ needed to resolve $M^{A}$ is very close to the optimal $L_{A}$.

\section{EXPERIMENTAL EVAluAtion OF REAL-TIME OPERATION}

This section describes an implementation of NCMA with real-time operation on a software-defined radio testbed. We also present experimental evaluation of the system.

\section{A. Implementation}

Hardware and Software Environment: Our NCMA implementation is built on the USRP/GNU Radio software radio platform. The USRP N210 (with SBX daughterboard) is connected to a PC (with Core i7 $3.4 \mathrm{GHz}$ and $16 \mathrm{G}$ RAM) through Gigabit Ethernet. The USRP hardware is equipped with a high-resolution GPS clock [8], and is driven by UHD software. We use UHD v003.005.003, and GNU Radio v3.6.5.

System Parameters: Our NCMA implementation adopts OFDM, with OFDM parameters (e.g., number of subcarriers, number of pilots) similar to those in 802.11. The data are BPSK modulated. At the PHY layer, we use a rate-1/2 convolutional code, and the Spiral soft-input Viterbi decoder [9]. We add an RS erasure code at the MAC layer to encode messages from the upper layer into MAC-layer packets, and to decode packets from the PHY layer into messages. The packet index with respect to the RS code is embedded into the MAC-layer packet header.

We implement the MUD decoder, the PNC decoder, and the RS decoder on GNU Radio. We use the default GNU Radio scheduler to perform baseband and MAC-layer processing. Thanks to the simplified design of UES in Sec. III, our current implementation can support real-time operation with $5 \mathrm{MHz}$ channel bandwidth. Each packet consists of 128 OFDM data symbols. Hence, the packet length is 384Bytes. We choose this packet length because the associated packet duration is approximately equal to the maximum packet duration in 20MHz 802.11 systems.

PHY-layer Challenges and Solutions: We modify the PHY frame formats of different user nodes, as illustrated in Fig. 6, so that they use orthogonal time-domain preambles and frequency-domain pilots for channel estimation and carrier frequency offset (CFO) tracking respectively. The data payloads of the two users, however, overlap in both time and subcarrier.

Large relative CFO may cause strong inter-carrier interference (ICI) [10]. In our current implementation, thanks to the accuracy of our GPS clocks [8], the relative CFO at the AP is in the ballpark of 200 to $300 \mathrm{~Hz}$ only, much smaller than the subcarrier bandwidth $78.125 \mathrm{KHz}$. Furthermore, the CFO stays constant after the oscillator has warmed up. As a result, ICI in our system is negligible. The small CFO, however, may still cause phase rotations in successive OFDM symbols. Phase rotations are tracked by pilots within the symbols and the varying channel coefficients caused by phase rotations are updated dynamically.

MAC-layer Challenges and Solutions: A major challenge in our design is that simultaneous transmissions must be synchronized to within the cyclic prefix (CP) of OFDM symbols (i.e., to within $3.2 \mu \mathrm{s}$ in our implementation). Doing so eliminates OFDM frequency-domain symbol misalignment 


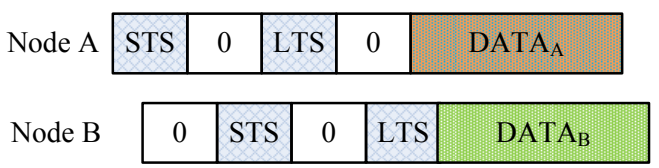

(a) Time domain

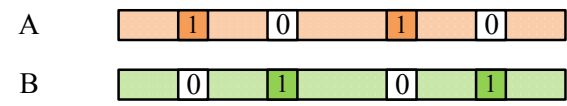

(b) Frequency domain

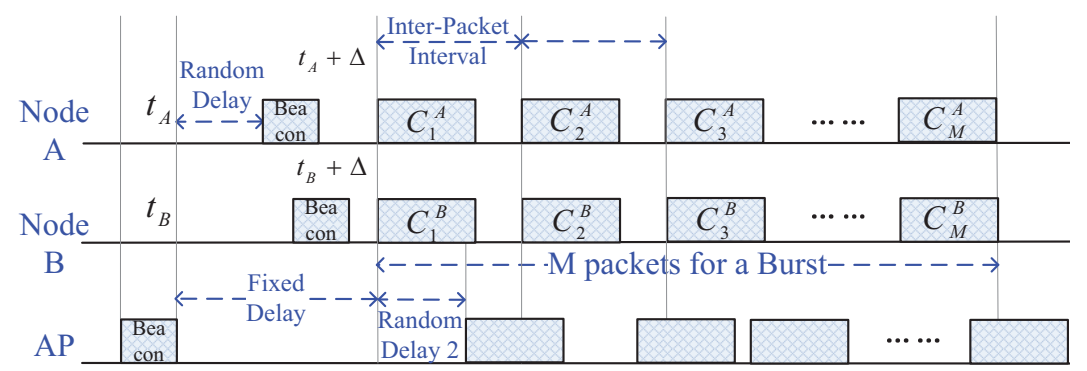

Fig. 7: NCMA MAC protocol: burst mode.

Fig. 6: Frame format for nodes A and B.

between users, and thus simplifies receiver design [10]. A possible approach is to leverage a reference packet (e.g., a beacon from the AP, or a downstream transmission from the AP) for time synchronization. That is, after decoding the reference packet and waiting for an inter-frame spacing, the two user nodes transmit together. However, the unpredictable random data-transfer and decoding latencies between the USRP and the PC (on the order of ms) invalidates this approach, which uses the time by which a received packet is decoded as a reference time. This is because the processing and decoding latencies at the two user nodes can be widely different [10]. To circumvent this problem, we adopt an approach that uses the time by which the preambles of a received packet are detected as a reference time, and adds an elastic delay at the two nodes so that they simultaneously transmit at a common fixed delay after the reception of a beacon frame from the AP. Specifically, in our design, the AP sends out beacon frames to trigger simultaneous transmissions by the two users. At the user nodes, the timestamp provided by the USRP FPGA hardware on the preamble detection time gives the beacon arrival time. The accuracy of the timestamp is on the order of ns. With beacon arrival time $t_{A}\left(t_{B}\right)$, node A (B) then times the transmission of its data frame to occur at $t_{A}+\Delta\left(t_{B}+\Delta\right)$. Here, $\Delta$ is a fixed time set to the maximum possible delay so as to remove the randomness of the data transfer time between the USRP and the PC, plus the randomness of the decoding and encoding processing times in the PC. In this way, the packets transmitted by nodes $\mathrm{A}$ and $\mathrm{B}$ are synchronized to within CP.

Now, if each beacon only triggers the synchronized transmissions of a pair of packet, then the above strategy will lead to much wastage because of the large overhead caused by the fixed delay, during which the wireless medium is idle. To reduce the overhead caused by the silent period, we adopt a burst mode in which one beacon triggers a burst of $N$ transmissions, as illustrated in Fig. 7. Also, to avoid overloading the processor of the USRP/GNU Radio platform with excessive packet processing (this will lead to overflow of the packets waiting to be processed at the AP), we add a gap between two successive transmissions within a burst to control the inter-packet interval (IPI) within the burst. Specifically, for the overall strategy, the first packet of each user node is transmitted at time $\Delta$ after the beacon arrival, and the transmissions of successive packets are spaced at IPI apart. In our current implementation, each beacon triggers 1,000 packet transmissions (i.e., $N=1,000$ ). The value of IPI is varied to test the system performance at various traffic loads.

One limitation of the USRP/GNURadio platform is the nonnegligible packet processing time, including bus transmission time, baseband processing time. As a result, real-time feedback such as ACK on a per-packet basis is not feasible. We let user nodes keep transmitting packets without packet-level ACK from the AP. The AP calls PHY-layer MUD and PNC decoders to decode packets, and invokes the RS message decoding algorithm when sufficient packets (equations) have been received that message decoding is possible.

\section{B. Experimental Setup}

We deployed six sets of USRP in an indoor office environment to simulate a WLAN system. The topology is shown in Fig. 8. Node 6 is the AP, and the other nodes are user nodes. The six USRPs are placed in different positions to create different channel conditions.

We performed two types of experiments: controlled and random. In the controlled experiments, we chose 3 USRPs, one as the AP and two as user nodes. We adjusted the transmission powers of the two user nodes to balance their SNRs. Here, the SNR is defined as the power of received preamble divided by the power of noise. The SNR averaged over all slots in each experiment is computed, and the performance result for this average SNR is presented. In the random experiments, we fixed the transmission powers of all nodes. We then chose two nodes for each experiment. All experiments were conducted at RF $2.585 \mathrm{GHz}$ without ISM band interference.

We experimented with the following four real-time systems:

(1) Single-User system ( $S U$ ): Only one user node is allowed to transmit at a time. Similar to our NCMA system and the MUD system below, beacons are used to trigger transmissions. Two user nodes transmit their packets in non-overlapping time slots.

(2) MUD system (MUD): Two user nodes transmit together. The receiver only adopts the MUD decoder. There is no PHY-layer bridging and MAC-layer bridging.

(3) NCMA with TES system (NCMA-TES): Two user nodes transmit together. The receiver adopts both the MUD decoder and the PNC decoder. MAC decoding based on TES is used.

(4) NCMA with UES system (NCMA-UES): Two user nodes transmit together. The receiver adopts both the MUD 


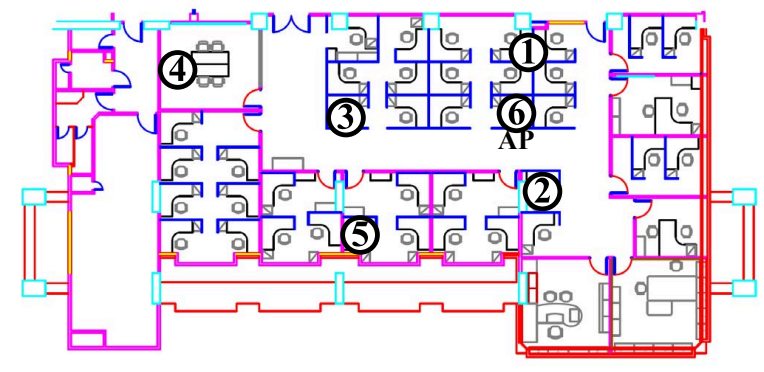

Fig. 8: Experimental testbed layout: there are six USRP nodes labeled as above. Node 6 is the AP. The other nodes are user nodes.

decoder and the PNC decoder. MAC decoding based on UES is used.

The MUD decoder and PNC decoder in the experiments here are implemented using a reduced-constellation scheme to speed up decoding. The reader is referred to Sec. V for details. We also remark that SU and MUD above also incorporate MAC-layer channel coding as in NCMA systems. Although the use of MAC-layer erasure codes does not improve the performance of SU and MUD, message-level ACK eases their implementations on USRP.

\section{Real-Time Experimental Evaluation}

To benchmark real-time performance, we use the following two metrics:

- Message processing time: The message processing time is defined as the time interval between the message arrival time (the arrival time of the first packet of the message) and the message decoded time (the time at which the message is successfully decoded). Note that the message processing time includes the packet transmission times (airtimes) and the intended IPI gap times. For an $(N, L)$ RS code and an IPI of $I$, the message processing time will be at least $L \times I$.

- Throughput: Let $N_{A}$ and $N_{B}$ be the numbers of messages of node $\mathrm{A}$ and node $\mathrm{B}$, respectively, that are delivered during an experiment of duration $T$. The throughput is defined as $\frac{\left(N_{A} \times L_{A}+N_{B} \times L_{B}\right) \times P}{T}$, where $P$ is the packet size in bits.

Message Processing Time: We adjusted the powers of the two user nodes to balance their SNR at 9dB. We used an $(255,16)$ RS code. Fig. 9 shows the trace of a burst of successive transmissions triggered by one beacon when the IPI=20ms, for NCMA-TES and NCMA-UES.

With respect to Fig. 9, our first observation is that as time goes by, the message processing time of NCMA-UES is almost constant while that of NCMA-TES system increases without bound. That is, the rate at which messages are processed in the former can keep up with the rate at which messages arrive, but not in the latter. This means that NCMA-UES can operate in real time while NCMA-TES cannot when IPI $=20 \mathrm{~ms}$. Our second observation is that NCMA-UES has throughput roughly twice that of NCMA-TES. The gradients

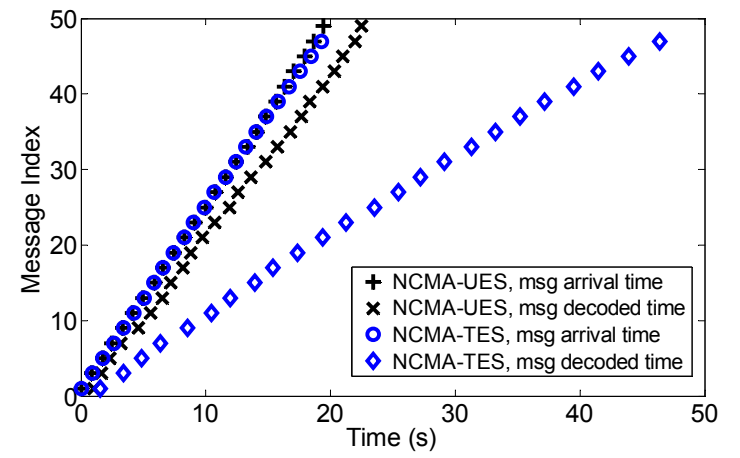

Fig. 9: Message decoding procedures of node A for NCMATES and NCMA-UES with IPI $=20 \mathrm{~ms}$.

of the two message decoded time curves in Fig. 9 correspond to the respective throughputs of the two systems.

Next we reduced IPI and measured the processing times of successive messages. Fig. 10 shows the results for NCMAUES, MUD and SU. When IPI=10ms, the message processing time of NCMA-UES keeps increasing, while for IPI $=20 \mathrm{~ms}$, it is almost constant. That is, IPI $=10 \mathrm{~ms}$ will saturate NCMAUES in the long run. Thus, we choose IPI $=20 \mathrm{~ms}$ to be the working IPI for further experiments of NCMA. A better MAClayer design, especially the use of a sparse erasure code, will be helpful to reduce the message processing time of NCMA II when IPI is small. We leave this as part of our future work.

Throughput: Fig. 11 shows the throughputs of different systems. We see that the performance of NCMA-UES is roughtly $100 \%$ higher than that of our previous design (i.e., NCMA-TES) at medium SNR (8dB-9dB). The improvement is even higher at medium-high SNR $(\geq 9 \mathrm{~dB})$. The main reason is that NCMA-TES takes much longer time for message processing, which lowers the data rate. The gain of NCMAUES over MUD is around $40 \%$ at $S N R=8.5 \mathrm{~dB}$. However, the gain over MUD diminishes as SNR increases, which is predictable since MUD works well starting from medium SNR.

We also compare the performance of NCMA-UES with SU. We tried two SU systems: one with IPI $=10 \mathrm{~ms}$, and one with IPI $=20 \mathrm{~ms}$. At IPI $=20 \mathrm{~ms}$ and $S N R \geq 8 \mathrm{~dB}$, we see that NCMA-UES outperforms SU by $100 \%$. At IPI $=10 \mathrm{~ms}$ and $S N R \leq 9 \mathrm{~dB}$, the gain of NCMA-UES over $\mathrm{SU}$ is not as much. We note, however, that NCMA-UES can achieve a higher throughput if it were not computation limited (i.e., if a more powerful processor were used), while SU cannot improve by much further. For example, if we consider the special case where IPI is adjusted so that there is no idle airtime in packet bursts, the performance of NCMA-UES will improve to $1.93 \mathrm{Mbps}$, while the performance of SU will only improve to $950 \mathrm{Kbps}$ (at $S N R=8 \mathrm{~dB}$ ).

\section{Computation-Unlimited Trace-Driven Analysis}

While Sec. IV-C investigated throughputs in computationlimited systems (as opposed to airtime or communications limited), here we are interested in the computation-unlimited throughputs - i.e., how would different schemes compare if 


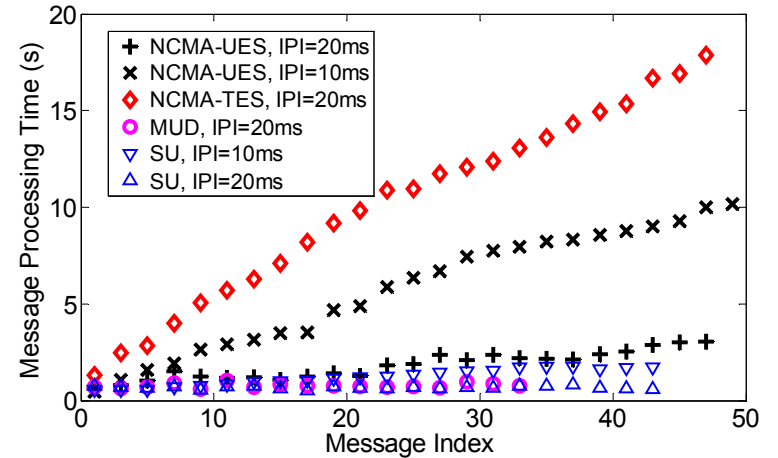

Fig. 10: Message processing time of node A for NCMA-UES, NCMA-TES, MUD, SU with different IPIs.

the signal processing and channel decoding speed were not the bottleneck? We consider only NCMA with UES and NCMA with TES here. In particular, the PHY-layer decoding statistics of these two systems are the same given the same PHY-layer decoders. The relative throughputs of these two systems then depend on how many PHY-layer packets need to be received before a MAC-layer message can be decoded.

Normalized Throughput: We define a new metric, normalized throughput, to compare different systems. Recall that we use beacon to trigger burst transmissions. We count the number of decoded messages belonging to node $\mathrm{A}$ and node $\mathrm{B}, N_{A}$ and $N_{B}$, respectively. The normalized throughput is $\frac{N_{A} \times L_{A}+N_{B} \times L_{B}}{N_{T X}}$, where $N_{T X}$ is the total number of PHYlayer packet transmissions. Obviously, the maximum normalized throughput for NCMA is 2 packets per time slot since our current system allows at most two users to transmit together. For SU, the maximum normalized throughput is 1 packet per time slot.

To measure normalize throughput, we adopt a trace-driven simulation approach. That is, instead of running a complete NCMA system, we disable NCMA decoders, and gather raw baseband samples provided by USRP. Then we use GNU Radio to perform offline processing on raw samples. In this way, we can easily compare different systems using the same trace.

In addition, with the same trace, we can calculate an upper bound of the normalized throughput. We count the number of equations received in each experiment. Let $E$ be the overall number of equations received and $T$ be the number of time slots. An upper bound is $\frac{E}{T}$. Since the number of equations per time slot is at most 2, the upper bound cannot exceed 2.

Effect of $L_{A}$ and $L_{B}$ on NCMA Systems: In NCMA, the RS code parameter $L$ can be different for different users, i.e., $L_{A}$ can be different from $L_{B}$. This asymmetric setup could improve the NCMA-TES system performance by much, because MAC-layer bridging could make better use of available XOR PHY packets as shown in [1]. Table II(a) shows the throughput of NCMA-UES and NCMA-TES for different ratio of $L_{A}$ and $L_{B}$ for $S N R_{A}=S N R_{B}=8 \mathrm{~dB}$ and $L_{B}=16$. To conserve space, we omit results that show similar behaviour for other ratios and SNRs. As can be seen from Table II(a), NCMA-TES is sensitive to ratio $\frac{L_{A}}{L_{B}}$ while NCMA-UES is

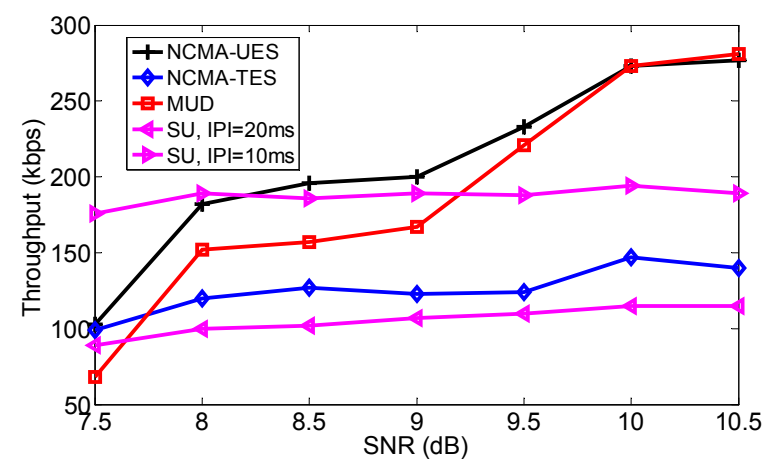

Fig. 11: Throughput comparison of different systems for different SNRs $\left(S N R_{A}=S N R_{B}\right)$ with $L_{A}=L_{B}=16$.

not. In particular, the maximum throughput can generally be obtained by NCMA-UES regardless of the setting of $\frac{L_{A}}{L_{B}}$. This phenomenon is predictable from our UES analysis in Sec. III-D.

Since NCMA-UES is not sensitive to asymmetric $\frac{L_{A}}{L_{B}}$, we next set $L_{A}=L_{B}=L$ and consider the effect of different $L$ on NCMA systems. Table II(b) shows the normalized throughputs for $S N R_{A}=S N R_{B}=8 \mathrm{~dB}$ with $L=16,32,64$. The results for other $L$ and other SNRs are similar. From Table II(b), we see that NCMA-UES is not sensitive to different $L$.

Being able to operate well for symmetric $\frac{L_{A}}{L_{B}}$ and different values of $L=L_{A}=L_{B}$ means we do not need to worry too much about optimizing and tuning these parameters in NCMA-UES. This simplifies its deployment and operation in practice.

Normalized Throughput: We performed two sets of experiments to compare system throughput under various SNRs. One is the balanced SNR case, and the other is the unbalanced SNR case. Node A and node B use the same $L=16$.

Fig. 12 shows the throughputs of different schemes for different balanced SNRs $\left(S N R_{A}=S N R_{B}\right)$. NCMA-UES outperforms NCMA-TES by $20 \%$, and outperforms MUD by $40 \%$ when $8 \mathrm{~dB} \leq S N R \leq 9.5 \mathrm{~dB}$. At $S N R \geq 9.5 \mathrm{~dB}$, the performance of NCMA-TES approaches NCMA-UES, since there are few lone XORs packets received in these SNR regimes-the difference between the two schemes comes from their respective MAC-layer bridging processes; without lone XOR packets, the decoding of the two user messages are decoupled and MAC-layer bridging will not come into effect. At $S N R \geq 10.5 \mathrm{~dB}$, NCMA-UES, NCMA-TES and MUD schemes converge to throughput of 2 .

\section{IMPROVING PERFORMANCE AT LOW SNR}

This section is devoted to improving NCMA performance in the low-SNR regime. As can be seen in Fig. 12, the performance of NCMA systems is lower than SU at low SNR (i.e., $7.5 \mathrm{~dB}$ ). This poor performance is due to our MUD decoder and PNC decoder designs. In this section, we investigate two new techniques that improve the performance of these PHYlayer decoders. 
TABLE II: Normalized throughput comparison of NCMA systems with (a) various ratios of $\frac{L_{A}}{L_{B}}$ and $L_{B}=16$; (b) various $L_{A}=L_{B}=L$ for $S N R_{A}=S N R_{B}=8 \mathrm{~dB}$.

(a)

\begin{tabular}{|c|c|c|c|}
\hline$\frac{L_{A}}{L_{B}}$ & NCMA-TES & NCMA-UES & Upper Bound \\
\hline \hline 1 & 0.746 & 0.916 & 0.929 \\
\hline 1.5 & 0.910 & 0.910 & 0.929 \\
\hline 2 & 0.913 & 0.913 & 0.929 \\
\hline
\end{tabular}

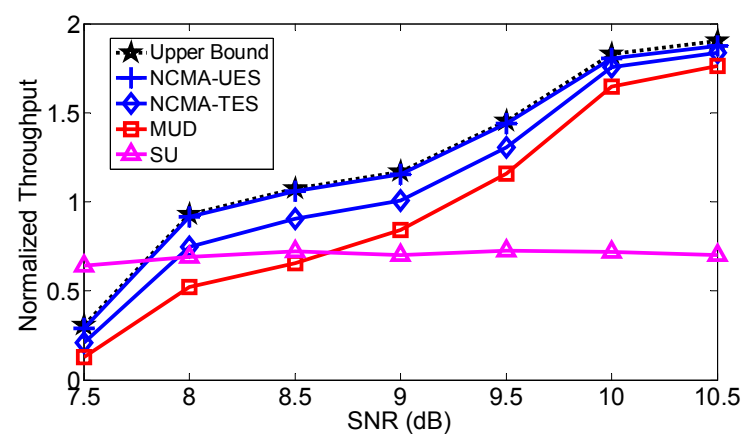

Fig. 12: Throughput comparison of different schemes for different SNRs $\left(S N R_{A}=S N R_{B}\right)$ with $L_{A}=L_{B}=16$.

\section{A. Overview of PHY-layer Decoders}

We first review our PHY-layer decoders. The PNC decoder attempts to decode $C_{i}^{A} \oplus C_{i}^{B}$, while the MUD decoder attempts to decode both $C_{i}^{A}$ and $C_{i}^{B}$. Our NCMA prototype assumes the use of the same convolutional code and the use of BPSK modulation at nodes $\mathrm{A}$ and $\mathrm{B}$.

PNC Decoder: We adopt the XOR-CD decoding approach for PNC decoding [5]. In XOR-CD, the overlapped received signals are first processed by a demodulator, which outputs symbol-by-symbol soft XOR information for feeding to a channel decoder to decode for packet $C_{i}^{A} \oplus C_{i}^{B}$. The essence of XOR-CD is explained as follows. For clarity, we drop the packet index $i$ here.

Let $X^{A}=\left(X_{A}[1], \ldots, X_{A}[k], \ldots\right)$ and $X^{B}=$ $\left(X_{B}[1], \ldots, X_{B}[k], \ldots\right)$ denote the PHY-layer modulated codewords (transmitted packets), and let $\Pi(\cdot)$ denote the convolutional coding operation. Convolutional codes are linear. Therefore, $\prod(\cdot)$ is linear, and we have $X^{A} \oplus X^{B}=$ $\prod\left(C^{A}\right) \oplus \prod\left(C^{B}\right)=\prod\left(C^{A} \oplus C^{B}\right)$, where $X^{A} \oplus X^{B} \triangleq$ $\left(X_{A}[k] \oplus X_{B}[k]\right)_{k=1,2, \ldots}$ is the bit-wise XOR of two packets, assuming $X_{A}[k]$ and $X_{B}[k]$ are binary symbols. This means we could first detect the XOR of individual bits $\left\{X_{A}[k] \oplus X_{B}[k]\right\}_{k=1,2 \ldots}$ and then feed these XOR bits to a standard convolutional decoder, such as the standard Viterbi decoder [1], for channel decoding.

MUD Decoder: For MUD decoding, the overlapped received signals are processed by two demodulators, which outputs symbol-by-symbol soft information on $X_{A}[k]$ and $X_{B}[k], k=1,2, \ldots$ for feeding to two separate channel decoders to decode for $C_{i}^{A}$ and $C_{i}^{B}$, respectively. In our implementation, the channel decoders in our MUD decoders and PNC decoder are the same: they both use the standard soft-input-hard-output Viterbi decoder originally designed for single-user communication [1]. The difference between them (b)

\begin{tabular}{|c|c|c|c|}
\hline$L$ & NCMA-TES & NCMA-UES & Upper Bound \\
\hline \hline 16 & 0.746 & 0.916 & 0.929 \\
\hline 32 & 0.744 & 0.908 & 0.929 \\
\hline 64 & 0.744 & 0.917 & 0.929 \\
\hline
\end{tabular}

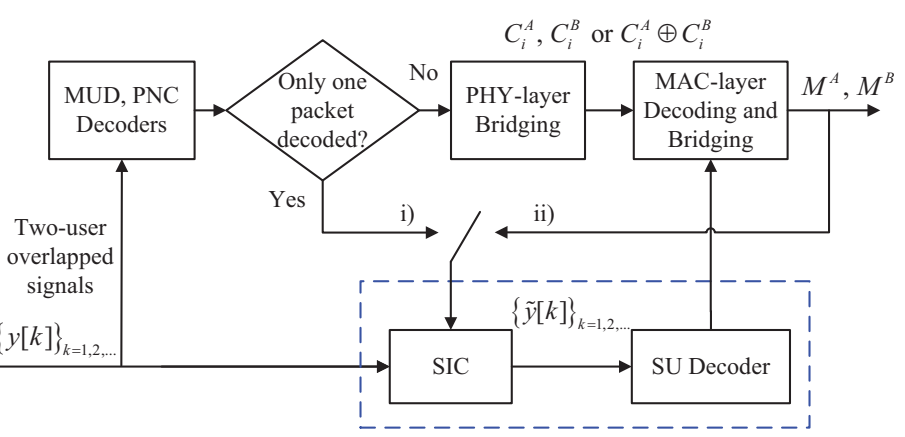

Fig. 13: Flowchart of NCMA II with NC-SIC PHY-layer decoder.

lie in the soft input information fed to the standard Viterbi decoder. That is, their demodulators are different.

Reduced-Constellation Demodulation: To be compatible with the standard binary Viterbi decoder, both the PNC demodulator and the MUD demodulator use a reduced-constellation demodulation technique to demodulate the received overlapped signals into soft information for $\left\{X_{A}[k] \oplus X_{B}[k]\right\}_{k=1,2, \ldots}$, $\left\{X_{A}[k]\right\}_{k=1,2, \ldots}$, and $\left\{X_{B}[k]\right\}_{k=1,2, \ldots}$, respectively.

The computation of the soft information for $X_{A}[k] \oplus X_{B}[k]$, $X_{A}[k]$, and $X_{B}[k]$, is similar for all $k$. For brevity, we will drop the bit index $k$ here. Let $y$ be the received signal. We have

$$
y=h_{A} x_{A}+h_{B} x_{B}+n,
$$

where $h_{A}$ and $h_{B}$ are channel gains of the received packets from nodes $\mathrm{A}$ and $\mathrm{B}$ respectively, and $n$ is Gaussian noise with variance $\sigma^{2}$.

In our design, the soft information of $x_{A} \oplus x_{B}$ is the $\log$ likelihood ratio (LLR):

$\log \frac{P_{0}}{P_{1}}=\log \frac{\operatorname{Pr}\left\{x_{A}=1, x_{B}=1 \mid y\right\}+\operatorname{Pr}\left\{x_{A}=-1, x_{B}=-1 \mid y\right\}}{\operatorname{Pr}\left\{x_{A}=-1, x_{B}=1 \mid y\right\}+\operatorname{Pr}\left\{x_{A}=1, x_{B}=-1 \mid y\right\}}$,

where $P_{0}=\operatorname{Pr}\left[x_{A} \oplus x_{B}=1 \mid y\right]$ and $P_{1}=\operatorname{Pr}\left[x_{A} \oplus x_{B}=\right.$ $-1 \mid y]$ (note that for BPSK, 1 represents the binary value of 0 and -1 represents the binary value of 1 ).

In the MUD demodulator associated with node A, for the soft information of $x_{A}$, instead of (17), we have

$\log \frac{P_{0}}{P_{1}}=\log \frac{\operatorname{Pr}\left\{x_{A}=1, x_{B}=1 \mid y\right\}+\operatorname{Pr}\left\{x_{A}=1, x_{B}=-1 \mid y\right\}}{\operatorname{Pr}\left\{x_{A}=-1, x_{B}=1 \mid y\right\}+\operatorname{Pr}\left\{x_{A}=-1, x_{B}=-1 \mid y\right\}}$,

where $P_{0}=\operatorname{Pr}\left[x_{A}=1 \mid y\right]$ and $P_{1}=\operatorname{Pr}\left[x_{A}=-1 \mid y\right]$.

Both (17) and (18) involve computation of the probabilities associated with the four constellation points: $\left(x_{A}, x_{B}\right)=$ $(1,1),(1,-1),(-1,1)$, and $(-1,-1)$. For further simplification, we could apply the log-max approximation to obtain 


$$
\begin{array}{r}
\log \frac{P_{0}}{P_{1}} \propto \max \left\{-\left|y-h_{A}-h_{B}\right|^{2},-\left|y+h_{A}+h_{B}\right|^{2}\right\}- \\
\max \left\{-\left|y+h_{A}-h_{B}\right|^{2},-\left|y-h_{A}+h_{B}\right|^{2}\right\}
\end{array}
$$

$$
\begin{array}{r}
\log \frac{P_{0}}{P_{1}} \propto \max \left\{-\left|y-h_{A}-h_{B}\right|^{2},-\left|y-h_{A}+h_{B}\right|^{2}\right\}- \\
\max \left\{-\left|y+h_{A}-h_{B}\right|^{2},-\left|y+h_{A}+h_{B}\right|^{2}\right\}
\end{array}
$$

for PNC and MUD associated with node A, respectively.

Essentially, (19) and (20) reduce the consideration of the four constellation points induced by the concurrent transmissions of nodes A and B to only two constellation points. The $\max \{\}$ operations in (19) and (20) can be considered as selecting the more likely constellation point between two choices in the argument of $\max \{\}$ to serve as the representative constellation point.

While (19) and (20) simplify the demodulation process, they are also "error-prone" in the low SNR regime. In particular, at low SNR, the two arguments within each $\max \{\}$ function may be of comparable magnitudes and the wrong choice can be easily made in the selection of the representative constellation point.

\section{B. Improving Performance at Low SNR with NC-SIC}

We leverage a common two-pass decoding technique, successive interference cancellation (SIC), to improve the accuracy of selecting constellation points at low SNR. We refer to the new design as NCMA with NC-SIC. Our NC-SIC exploits SIC by two means: PHY-layer SIC or cross-layer SIC, as explained below.

PHY-layer SIC: To explain PHY-layer SIC, let us suppose that only $C_{i}^{B}$ is decoded in the first pass. This means we will be able to get $X_{B}[k]$ for all $k$. With reference to (20), instead of $\max \{\}$, which is error-prone in the low SNR regime in that the wrong choice may be made, we now know exactly which argument in the $\max \{\}$ is the valid one. For a particular $k$, suppose that $X_{B}[k]=1$. Then $\log$ likelihood ratio for bit $k$ in the decoding of $C_{i}^{A}$ in the second pass is

$$
\log \frac{P_{0}}{P_{1}} \propto-\left|y-h_{A}-h_{B}\right|^{2}+\left|y+h_{A}-h_{B}\right|^{2} .
$$

The decoding based on (21) will be freed of the potential mistake that can be made by the $\max \{\}$ operation.

Similarly, suppose that instead of $C_{i}^{B}$, only $C_{i}^{A \oplus B}$ is decoded in the first pass, and that for a particular $k, X_{A}[k] \oplus$ $X_{B}[k]=1$. Then, the $\log$ likelihood ratio for bit $k$ in the decoding of $C_{i}^{A}$ in the second pass is

$$
\log \frac{P_{0}}{P_{1}} \propto-\left|y-h_{A}-h_{B}\right|^{2}+\left|y+h_{A}+h_{B}\right|^{2} .
$$

Cross-layer SIC: For cross-layer SIC, the lone packet whose signal is to be cancelled comes from the MAC-layer decoder. Suppose that one of the user messages, say $M^{B}$, is decoded at the MAC layer first. This means that $C_{i}^{B}$ for all $i$ are available; and for each $i$, the corresponding $X_{B}[k] \forall k$ are available. Suppose that $M^{A}$ cannot be decoded at the same time (even after MAC-layer bridging.). Then, we resort to cross-layer SIC to decode more PHY packets of type $C_{i}^{A}$. Specifically, the corresponding PHY-layer SIC as embodied in (21) can be used to decode $C_{i}^{A}$ if $C_{i}^{A}$ failed to be decoded in the previous round of PHY-layer decoding. Note that in the NCMA systems in this paper, we use dissimilar channel codes for nodes A and B. Therefore we cannot decode the XOR message in the strict form of $M_{A} \oplus M_{B}$, which is required to get $C_{i}^{A \oplus B} \forall i$. Thus, we cannot use SIC in the form of (22) for cross-layer SIC.

With the new SIC components, we modify flow of the decoding process as shown in Fig. 13. In addition to previous blocks in NCMA, we add SIC processing followed by an SU decoder. If the PHY layer decodes one and only one packet, we invoke the SIC-SU decoder block; if the MAC-layer UES decoder manages to recover PHY packets for time slots in which no packets were previously decoded by the PHY layer, we also invoke the SIC-SU decoder block. The packet decoded by the SIC-SU decoder block is fed to the MAC-layer UES decoder for further processing.

\section{Trace-Driven Simulation Results}

Experimental Setup: We have not implemented a realtime NCMA system with PHY-layer SIC and cross-layer SIC capability. Here, we use trace-driven simulations to compare it with other systems. We compare the following systems:

(1) $S U$ : This is the same SU system as in Sec. IV-B.

(2) MUD: This is the same MUD system as in Sec. IV-B.

(3) NCMA I: This is one of the NCMA systems proposed in [1]. In addition to the PNC decoder, two types of MUD decoders are concurrently used: reduced-constellationbased decoders, and SIC-based decoders. The outputs of these MUD decoders are combined to generate PHYlayer outcomes. MAC decoding based on TES is used.

(4) NCMA II with NC-SIC: This is the NCMA system defined in Sec. V-B. The MUD and PNC decoders are based on reduced-constellation. PHY-layer SIC and cross-layer SIC are added in the PHY layer. MAC decoding based on UES is used.

The trace-driven simulation results will demonstrate that the use of NC-SIC in NCMA (i.e., NCMA II with NC-SIC) leads to performance improvement over all other systems, including the NCMA-UES system proposed in Section IV. The improvement is especially pronounced at low SNR. The cost of this improvement, however, is increased processing complexity. The implementation details of NC-SIC will be discussed in Section VI.

Normalized Throughput of Balanced SNRs: We first present experimental results for controlled experiment. We used the same trace collected in Sec. IV-D. Since the throughput of NCMA I is sensitive to $L_{A}$ and $L_{B}$, we vary $L_{A}$ and $L_{B}$ (i.e., $L_{A} / L_{B}=[1,2,3,4]$, and $L_{B}=[8,16,32,64]$ ), and select the best throughput over all combinations as the final result.

Fig. 14 shows the normalized throughput of different systems for different SNRs. The throughput of NCMA II with NC-SIC is 1.5 times better than that of NCMA I at $S N R \leq$ $9 d B$. These results show that NCMA II with NC-SIC improves 


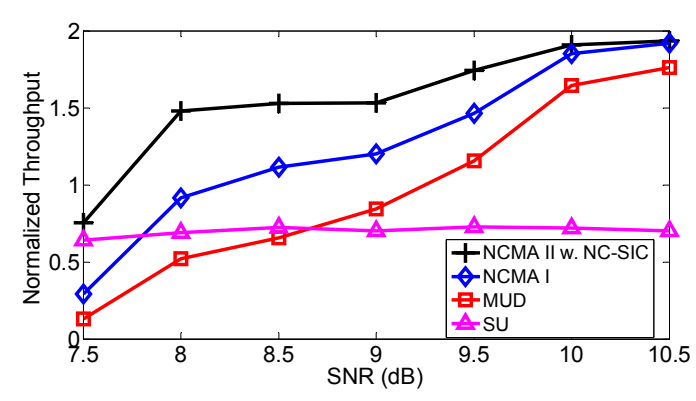

Fig. 14: Throughput comparison of different systems for different SNRs $\left(S N R_{A}=S N R_{B}\right)$.

NCMA performance not only at low SNR but also at medium SNR. In addition, compared with Fig. 12, at low SNR (e.g., $7.5 \mathrm{~dB}$ and $8 \mathrm{~dB}$ ), the throughput of NCMA II with NC-SIC surpasses that of SU. That is, NCMA II with NC-SIC makes the performance of NCMA comparable to SU at low SNR.

Normalized Throughput of Unbalanced SNRs: We next present the experimental results for unbalanced SNRs in random network topologies. We collected traces from the deployed nodes shown in Fig. 8. We form several user pairs as shown in Fig. 15. Users within a pair transmit together to the AP. Fig. 16 shows the throughputs of different systems. We present the throughputs of nodes A and B separately. For pair P2, both nodes are at medium SNR. The throughputs of NCMA I and NCMA II with NC-SIC are better than that of MUD. Pair P5, with node A at high SNR and node B at low SNR, exhibits similar results. For pair P3, although node B is at low SNR, the two NCMA systems can still improve its performance compared with MUD. For pair P4, while the throughput of NCMA II with NC-SIC is slightly smaller than that of SU, it is significantly better than that of NCMA I. This indicates the PHY-layer SIC and cross-layer SIC (i.e., two-pass processing) can improve NCMA performance substantially at low SNR.

Effects of NCMA II with NC-SIC on User Pairing: Here, we show that an appropriate user pairing strategy can improve the performance of nodes with low SNR. Suppose that nodes $2,3,4,5$ want to send messages to the AP. We compare two user pairing strategies for the NCMA II with NC-SIC system. In Strategy 1, we form user pairs P2 and P4: P2 pairs two strong users and P4 pairs two weak users; in Strategy 2, we form users pairs $\mathrm{P} 3$ and $\mathrm{P} 5$ : both $\mathrm{P} 3$ and $\mathrm{P} 5$ pair a strong user and a weak user. Note that both strategies include the same set of user nodes at Location 2, 3, 4 and 5; just that the pairing strategies are different. As shown in Fig. 17, the overall system throughput of Strategy 2 is $40 \%$ higher than that of Strategy 1. In particular, Strategy 2 improves the throughputs of weak users at Locations 4 and 5 significantly, without degrading the throughputs of the strong users at Locations 2 and 3 by much.

Overall, our investigation of NCMA performance at low SNR points to two performance-improvement strategies that can be used in conjunction: (i) NC-SIC; (ii) User pairing.

\section{DISCUSSION}

In this section, we discuss the complexity and implementation of NC-SIC. In addition, we explain how to extend the

\begin{tabular}{|c|c|c|}
\hline User Pair & User A & User B \\
\hline \hline P1 & Location 1 (20dB) & Location 2 (12.3dB) \\
\hline P2 & Location 2 (12.3dB) & Location 3 (9dB) \\
\hline P3 & Location 3 (9dB) & Location 4 (7dB) \\
\hline P4 & Location 4 (7dB) & Location 5 (7.4dB) \\
\hline P5 & Location 2 (12.3dB) & Location 5 (7.4dB) \\
\hline
\end{tabular}

Fig. 15: User pairing in random topology. framework presented thus far to NCMA with more than two users.

Complexity of NC-SIC: The preceding section showed that NC-SIC can significantly improve the performance of NCMA. Recall that for NC-SIC, we first decode a correct packet from the PHY-layer decoders and/or MAC-layer decoder, and then leverage this decoded packet information to improve the accuracy of selecting constellation points. Such two-pass decoding does add complexity to the overall system. Let us consider the complexities of PHY-layer SIC and cross-layer SIC separately.

In addition to the first-pass decoding, PHY-layer SIC leverages the decoded packet to perform decoding again in the second pass. Note that there are three parallel decoders in the first pass, and the PHY-layer SIC only needs one decoder. Thus, the relative increase of the complexity at the PHY layer is $1 / 3$. PHY-layer SIC may give more equations to the MAC layer per time slot. However, it will not increase the complexity of message decoding at the MAC layer, since message decoding in NCMA II is based on UES, for which PHY-layer SIC does not introduce more MAC-layer operations. Also, note that the second pass is only invoked in time slots for which only one packet is decoded. Therefore, PHY-layer SIC will only increase the complexity by $1 / 3$ in the worst-case scenario.

Cross-layer SIC is invoked when the message of one of the users, say user A, is decoded at the MAC layer. The MAC layer decoder first recovers packets associated with message A in time slots for which no packets (A's packets, B's packets, or XOR packets) were decoded at the PHY layer. The packet recovery is basically a form of packet re-encoding based on message A. Then, PHY-layer SIC is performed to remove the signal associated with packet $\mathrm{A}$ in each of the time slots. After that, attempts are made to decode the packets of user B based on the remaining signal. As can be seen from the above, both the MAC layer and the PHY layer incur increased complexity as a result of cross-layer SIC.

To dig deeper into the increased complexity, suppose that user A, whose message has been decoded, uses an $\left(N, L_{A}\right)$ RS code, and user B uses an $\left(N, L_{B}\right)$ RS code. In the worst-case scenario, user A transmits $N$ packets before the AP decodes $L_{A}$ of the packets; furthermore, in all of these $N$ time slots, no user B's packets and no XOR packets are decoded. In this case, in the first pass, the PHY layer attempts to decode 


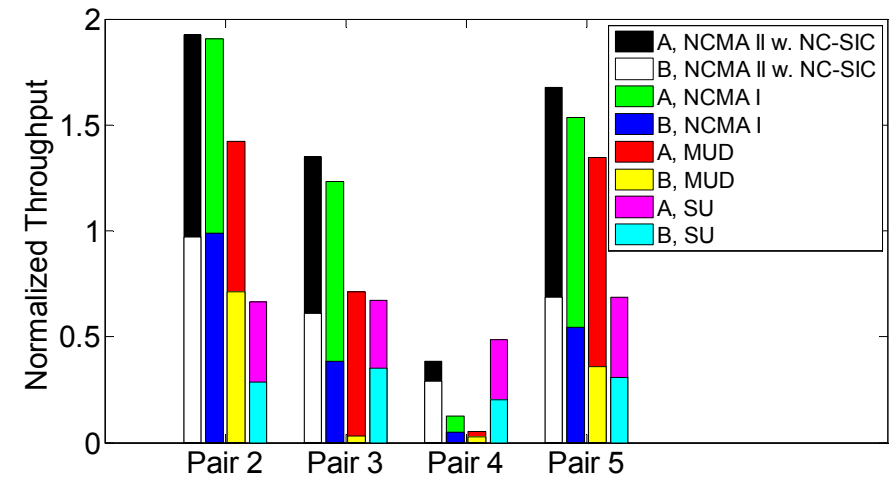

Fig. 16: Throughputs of five user pairs in Fig. 15 under NCMA II (NC-SIC), NCMA I, MUD and SU.

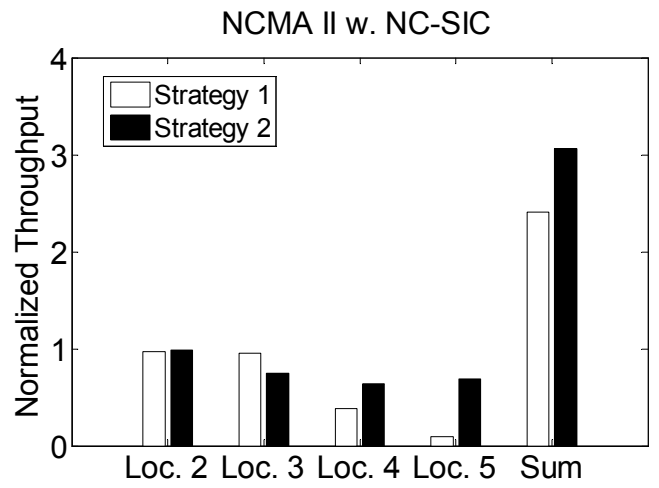

Fig. 17: Throughputs of two different user-pairing strategies under NCMA II. user A's packets, user B's packets and XOR packets in the $N$ time slots using three parallel PHY-layer decoders. The PHYlayer complexity is therefore of order $3 N$. In addition, in the first pass, the MAC layer solves user A's message using the $L_{A}$ decoded packets of user A. Recall that this is to solve equation $\tilde{G}^{A} M^{A}=\tilde{C}^{A}$, where the sizes of $\tilde{G}^{A}, M^{A}$, and $\tilde{C}^{A}$ are $L_{A} \times L_{A}, L_{A} \times K, L_{A} \times K$, respectively, and $K$ is the length of PHY-layer packets (See Section III-A). Assume that addition or multiplication of individual elements has complexity $\mathcal{O}(1)$. The complexity of inverting $\tilde{G}^{A}$ is $\mathcal{O}\left(L_{A}{ }^{3}\right)$, and the complexity of calculating $M^{A}$ by $\left(\tilde{G}^{A}\right)^{-1} \tilde{C}^{A}$ is $\mathcal{O}\left(L_{A}^{2} K\right)$. The MAC-layer complexity for the first pass is therefore $\mathcal{O}\left(L_{A}{ }^{3}+L_{A}{ }^{2} K\right)$. The first pass is common to both the scheme with and the scheme without cross-layer SIC. Now, only the scheme with cross-layer has a second pass. For the second pass, the MAC layer first recovers $N-L_{A}$ user A's packets. The recovery operations are to multiply the corresponding $N-L_{A}$ rows of the generator matrix $G_{A}$ by $M^{A}$. This is to multiply an $\left(N-L_{A}\right) \times L_{A}$ matrix by an $L_{A} \times K$ matrix. Assume that $G_{A}$ can be calculated beforehand, then this calculation does not add complexity. The MAC-layer complexity of the second pass is therefore $\mathcal{O}\left(\left(N-L_{A}\right) L_{A} K\right)$. After that, the PHY layer attempts to decode $N-L_{A}$ user B's packets by PHY-layer SIC. The PHY-layer complexity of the second pass is therefore of order $N-L_{A}$. Compared with the scheme without cross-layer SIC, the relative increase in complexity is the complexity of the second pass divided by the complexity of the first pass. Hence, the relative increase in complexity at the PHY layer is $\left(N-L_{A}\right) / 3 N$, and the relative increase in the complexity at the MAC layer is $\mathcal{O}\left(\left(N-L_{A}\right) K /\left(L_{A}{ }^{2}+L_{A} K\right)\right)=\mathcal{O}\left(\left(N-L_{A}\right) / L_{A}\right)$.

Real-time Implementation of NC-SIC: Real-time implementation of NC-SIC is feasible on USRP/GNU Radio. In the current OFDM implementation of GNU Radio, there are several important blocks: frame synchronization, sampler, fast Fourier transform (FFT), demodulation, channel decoding and framing. These blocks are cascaded in a linear fashion with the output of one block feeding to the input of the next block. The baseband samples from USRP are passed between the successive blocks as data streams. In particular, the baseband samples are first passed to the frame synchronization block. Upon the detection of a packet, the frame synchronization block will alert the following blocks to get to work. The last block in the chain, the framing block, delivers the decoded packet to the MAC layer.

To implement NC-SIC, we need to change the conventional OFDM implementation. Specially, for those time slots for which no packets are successfully decoded, we need to store input samples at the demodulation block. If PHY-layer SIC or cross-layer SIC is invoked, we perform demodulation again with these stored samples.

A concern is that the increased computation complexity may further increase the already-large packet decoding latency on the USRP/GNU Radio platform. To reduce latency, we can move some computation-intensive signal processing operations before demodulation, such as frame synchronization, sampler and FFT, to field programmable gate arrays (FPGAs) in USRP. The real-time implementation of NC-SIC will be left as part of our future work.

NCMA for More Than Two User Nodes: In general, NCMA may allow simultaneous transmissions by more than two users. The principles of UES, reduced-constellation demodulation and NC-SIC remain valid with minor modifications. Without loss of generality, let us focus on the case of three user nodes for concreteness (the three-user example illustrates the generalization process).

Let the set of user nodes be denoted by $\Theta=\{A, B, C\}$. Suppose that the AP receives overlapped signals from the three user nodes. Let $C_{i}^{s}, s \in \Theta$, be the data packet transmitted by user node $s$ in time slot $i$. Three MUD decoders are used to decode $C_{i}^{s}$, where $s \in \Theta$. Three PNC decoders are used to decode $C_{i}^{s} \oplus C_{i}^{s^{\prime}}$, where $s \neq s^{\prime} \in \Theta$, and one PNC decoder is used to decode $C_{i}^{A} \oplus C_{i}^{B} \oplus C_{i}^{C}$. In short, there are seven decoders, each outputs a linear equation of the form $a C_{i}^{A} \oplus$ $b C_{i}^{B} \oplus c C_{i}^{C}$, where $a, b, c \in\{0,1\}$ and not all $a, b, c$ are zeroes.

Consider the outputs of the seven decoders. In general, only a subset of the linear combinations are successfully decoded and delivered to the MAC layer. Altogether, there are $2^{7}$ possible subsets. However, some of these subsets are "equivalent". For example, the subsets $\left\{C_{i}^{A}, C_{i}^{A} \oplus C_{i}^{B} \oplus C_{i}^{C}\right\}$ and $\left\{C_{i}^{A}, C_{i}^{B} \oplus C_{i}^{C}\right\}$ are equivalent, because by PHY-layer bridging, we can reduce $C_{i}^{A} \oplus C_{i}^{B} \oplus C_{i}^{C}$ in the first subset to $C_{i}^{B} \oplus C_{i}^{C}$ with the information of $C_{i}^{A}$. Overall, these $2^{7}$ possibilities can be reduced to the following eight non- 
equivalent scenarios [11]:

1) The MAC layer receives only one native packet $C_{i}^{s}$, where $s \in \Theta$

2) The MAC layer receives two native packets $C_{i}^{s}$ and $C_{i}^{s^{\prime}}$, where $s \neq s^{\prime} \in \Theta$

3) The MAC layer receives all three native packets $C_{i}^{A}$, $C_{i}^{B}$ and $C_{i}^{C}$;

4) The MAC layer receives only one XOR of the form $C_{i}^{s} \oplus C_{i}^{s^{\prime}}$, where $s \neq s^{\prime} \in \Theta$

5) The MAC layer receives the XOR packet of the form $C_{i}^{A} \oplus C_{i}^{B} \oplus C_{i}^{C}$;

6) The MAC layer receives one XOR packet $C_{i}^{s} \oplus C_{i}^{s^{\prime}}$, and one native packet $C_{i}^{s^{\prime \prime}}$, where $s \neq s^{\prime} \neq s^{\prime \prime} \in \Theta$;

7) The MAC layer receives two XOR packets $C_{i}^{A} \oplus C_{i}^{B}$ and $C_{i}^{B} \oplus C_{i}^{C}$ (note that the subsets $\left\{C_{i}^{A} \oplus C_{i}^{B}, C_{i}^{A} \oplus C_{i}^{C}\right\}$, $\left\{C_{i}^{A} \oplus C_{i}^{C}, C_{i}^{B} \oplus C_{i}^{C}\right\}$ and $\left\{C_{i}^{A} \oplus C_{i}^{B}, C_{i}^{A} \oplus C_{i}^{C}, C_{i}^{B} \oplus\right.$ $\left.C_{i}^{C}\right\}$ are all equivalent to the subset $\left\{C_{i}^{A} \oplus C_{i}^{B}, C_{i}^{B} \oplus\right.$ $\left.C_{i}^{C}\right\}$ chosen as the representative here);

8) The MAC layer receives no packet.

With these eight outcomes, for message-level decoding at the MAC layer, we can build a unified equation system in a similar way as the two-user system, and establish similar conditions for successful message decoding.

For the implementation of the PHY-layer decoders for the three-user system, we can also extend the reducedconstellation demodulation strategy used in the two-user system. Take the PNC decoding of $C_{i}^{A} \oplus C_{i}^{B}$ for example. The soft information of $X_{A}[k] \oplus X_{B}[k]$ for a particular bit index $k$ is the LLR

$$
\begin{aligned}
\log \frac{P_{0}}{P_{1}}=\log \frac{\operatorname{Pr}\left\{x_{A} \oplus x_{B}=1, x_{C}=1 \mid y\right\}}{\operatorname{Pr}\left\{x_{A} \oplus x_{B}=-1, x_{C}=1 \mid y\right\}} \\
\frac{+\operatorname{Pr}\left\{x_{A} \oplus x_{B}=1, x_{C}=-1 \mid y\right\}}{+\operatorname{Pr}\left\{x_{A} \oplus x_{B}=-1, x_{C}=-1 \mid y\right\}},
\end{aligned}
$$

where $x_{A}, x_{B} \in\{-1,1\}$ represent the modulated signals of $X_{A}[k], X_{B}[k] \in\{0,1\}$ (we drop the bit index $k$ here and below for brevity). Then we could apply the log-max approximation as in the two-user system to simplify the computation.

At low SNR, PHY-layer SIC and cross-layer SIC can also be applied. For PHY-layer SIC, let us illustrate with three representative cases below (the general principle can be extrapolated from the specific cases below):

a) If $C_{i}^{A}$ is decoded in the first pass, and $X_{A}[k]=1$ for a particular index $k$, then this case can be reduced to the case of two user nodes B and C for NCMA after SIC. The cases where $C_{i}^{B}$ or $C_{i}^{C}$ is decoded first are similar;

b) If $C_{i}^{A} \oplus C_{i}^{B}$ is decoded in the first pass, and $X_{A}[k] \oplus$ $X_{B}[k]=1$ for a particular index $k$, then the LLR for bit $k$ in the decoding of $C_{i}^{A}$ in the second pass is

$$
\begin{aligned}
\log \frac{P_{0}}{P_{1}}=\log \frac{\operatorname{Pr}\left\{x_{A}=1, x_{B}=1, x_{C}=1 \mid y\right\}}{\operatorname{Pr}\left\{x_{A}=-1, x_{B}=-1, x_{C}=1 \mid y\right\}} \\
\frac{+\operatorname{Pr}\left\{x_{A}=1, x_{B}=1, x_{C}=-1 \mid y\right\}}{+\operatorname{Pr}\left\{x_{A}=-1, x_{B}=-1, x_{C}=-1 \mid y\right\}} .
\end{aligned}
$$

The decodings of $C_{i}^{B}, C_{i}^{C}, C_{i}^{B} \oplus C_{i}^{C}$ and $C_{i}^{A} \oplus C_{i}^{B} \oplus C_{i}^{C}$ are similar. The cases where $C_{i}^{B} \oplus C_{i}^{C}$ or $C_{i}^{A} \oplus C_{i}^{C}$ is decoded first are similar.

c) If $C_{i}^{A} \oplus C_{i}^{B} \oplus C_{i}^{C}$ is decoded in the first pass, and
$X_{A}[k] \oplus X_{B}[k] \oplus X_{C}[k]=1$ for a particular index $k$, then the LLR for bit index $k$ in the decoding of $C_{i}^{A}$ in the second pass is

$$
\begin{aligned}
\log \frac{P_{0}}{P_{1}}=\log & \frac{\operatorname{Pr}\left\{x_{A}=1, x_{B}=1, x_{C}=1 \mid y\right\}}{\operatorname{Pr}\left\{x_{A}=-1, x_{B}=-1, x_{C}=1 \mid y\right\}} \\
& \frac{+\operatorname{Pr}\left\{x_{A}=1, x_{B}=-1, x_{C}=-1 \mid y\right\}}{+\operatorname{Pr}\left\{x_{A}=-1, x_{B}=1, x_{C}=-1 \mid y\right\}} .
\end{aligned}
$$

The decodings of $C_{i}^{B}, C_{i}^{C}, C_{i}^{A} \oplus C_{i}^{B}, C_{i}^{B} \oplus C_{i}^{C}$ and $C_{i}^{A} \oplus C_{i}^{C}$ are similar.

For cross-layer SIC, suppose we decode one of the user messages, say $M^{A}$, first. This means that $C_{i}^{A}$ for all $i$ are available. Then, we can resort to the above case a) of PHYlayer SIC to decode more PHY-layer packets.

\section{RELATED WORK}

Concurrent Transmission Systems: NCMA is a system in which multiple users use the same signal space. By contrast, the users in traditional frequency-division multiple access (FDMA), time-division multiple access (TDMA), and codedivision multiple access (CDMA) systems use orthogonal or near-orthogonal (in the case of CDMA) signal space.

Although NCMA is an OFDM system, it is totally different from OFDMA wherein different users transmit their signals on different subcarriers. In conventional OFDMA, the subcarriers are either interleaved (e.g., [12]) or grouped into subgroups (e.g., [13]) for assignment to different users. In NCMA, multiple users use the same set of subcarriers when they transmit concurrently. Thus, the signals overlap in both frequency and time. This design improves spectrum efficiency.

Others have also proposed and demonstrated systems in which the signals of multiple users overlap in frequency and time. In [14], two users may use the same subcarriers. However, at least some subcarriers must be non-overlapping to bootstrap successive interference cancellation for packet recovery. Similarly, as a time-domain WLAN system, the set-up in [15] requires some payload symbols to be non-overlapping in time to bootstrap the packet decoding algorithm. NCMA, on the other hand, does not require non-overlapped time or frequency payload symbols to trigger the decoding process.

MAC Channel: From the information theoretical viewpoint, the channel from the multiple users to the AP in NCMA is a Multiple-Access Channel (MAC channel) [16], [17]. More specifically, it is an asynchronous MAC channel [18]. Ref. [19] pointed out that, for systems in which simultaneously transmitting users adopt the same waveform, symbol asynchrony has no effect on the two-user capacity region of the white Gaussian channel. Our paper here, on the other hand, is an experimental paper that investigates the asynchronous fading MAC channel in a real wireless environment. Rather than pursuing the ultimate capacity, we opt for simple decoder designs amenable to fast decoding algorithms. It is in this context that PNC decoding becomes useful as a complement to MUD decoding.

Network Coding for Wireless Networks: Network coding (NC) has been studied and implemented in wireless networks at the PHY layer [3], [4], and the network layer [20], [21]. 
However, the previous studies of $\mathrm{NC}$ have generally been restricted to relay networks, where $\mathrm{NC}$ was originally shown to be helpful for packet exchange via relays. NCMA, on the other hand, targets the non-relay setting, i.e., multiple access in wireless networks.

Recently, there have been some efforts in applying NC to multiple access in wireless networks. Besides our previous report on NCMA [1], [22], [23] and [24] explored how to resolve collisions among packets of different users. Instead of discarding the collided packets, a set of linear equations are formed to exploit information contained in them. Unlike NCMA, the decoding in [22]-[24] is based on PHY-layer equations only. In addition, their decoding methods are either pure MUD or pure PNC methods. By contrast, NCMA makes use of both MUD and PNC decoding at the PHY layer in a complementary way (using PHY-layer bridging); adding to that, it introduces another layer of MAC channel coding to introduce correlations among PHY-layer packets to obtain further performance gain (using MAC-layer bridging).

Advanced PHY-layer Techniques: Recently, interference cancellation techniques have been advanced and applied to the decoding of PHY-layer rateless codes [25], [26]. Distributed multiple-input multiple-output (MIMO) [27], [28] can enable spatially separated transmitters to form a virtual MIMO system for multiple access. For these techniques, the processing is entirely on signal samples rather than on bits. While having good performance, the decoding procedure could incur considerable storage and computation costs. NCMA opts for reduced complexity - i.e., simple PHY-layer decoding with real-time performance. The correlations among different PHY packets are exploited in MAC-layer decoding, which deals with bits rather than samples.

\section{CONCLUSIONS}

We have presented and experimentally evaluated an improved NCMA system, NCMA II. A number of new designs have been put forth and implemented into NCMA II. Specifically, NCMA II exploits a new unified framework for MAClayer decoding that enables real-time operation with higher throughput. In addition, NCMA II adopts new PHY-layer decoding techniques to improve performance at low SNR. Furthermore, a new MAC protocol to synchronize bursts of packet transmissions from multiple users has been implemented to alleviate the throughput bottleneck due to the large turnaround time between the USRP and PC in our software-radio prototype. The new designs and techniques put forth in this paper are crucial elements that move NCMA a step toward practicality. Going forward, two interesting directions are

- Sparse Codes for NCMA - NCMA II system makes use of an RS code for MAC layer channel coding. Sparse erasure codes, e.g., fountain codes, sparse random linear network codes, can potentially reduce the computation complexity further. An interesting question is whether sparse codes for NCMA should be designed differently than the sparse codes for conventional single-user system, and if so, how?

- High SNR Regime - At high SNR, using higher order modulations (e.g., QPSK and higher-order QAM) can improve throughput further. Also, currently NCMA II allows at most two users to transmit together. The theoretical framework of NCMA can potentially be generalized to more than two simultaneous transmissions. When SNR is high, implementation of such generalization can further raise the achievable throughput.

\section{REFERENCES}

[1] L. Lu, L. You, and S. C. Liew, "Network-coded multiple access," IEEE Transactions on Mobile Computing, 2014.

[2] S. Verdú, Multiuser Detection. Cambridge University Press, 1998.

[3] S. Zhang, S. C. Liew, and P. P. Lam, "Hot topic: physical-layer network coding," in ACM MOBICOM, 2006.

[4] P. Popovski and H. Yomo, "The anti-packets can increase the achievable throughput of a wireless multi-hop network," in IEEE ICC, 2006.

[5] S. Liew, S. Zhang, and L. Lu, "Physical-layer network coding: Tutorial, survey, and beyond," Physical Communication, vol. 6, no. 1, pp. 4-42, 2013.

[6] J. He and S. C. Liew, "Building blocks of physical-layer network coding," in IEEE SECON, 2013.

[7] C. Cooper, "On the distribution of rank of a random matrix over a finite field," in Random Struct. Algorithms, 2000.

[8] Ettus Inc., "Universal software radio peripheral."

[9] Spiral Project, "Viterbi decoder software generator," available: www.spiral.net/software/viterbi.html.

[10] L. Lu, L. You, Q. Yang, T. Wang, M. Zhang, S. Zhang, and S. C. Liew, "Real-time implementation of physical-layer network coding," in $A C M$ SRIF, 2013, pp. 71-76.

[11] S. Yang, S. C. Liew, L. You, and Y. Chen, "Linearly-coupled fountain codes for network-coded multiple access," in IEEE ITW, 2014.

[12] H. Sari and G. Karam, "Orthogonal frequency-division multiple access and its application to CATV networks," European transactions on telecommunications, vol. 9, no. 6, pp. 507-516, 1998

[13] K. Tan, J. Fang, Y. Zhang, S. Chen, L. Shi, J. Zhang, and Y. Zhang, "Fine-grained channel access in wireless LAN," in ACM SIGCOMM, 2010.

[14] L. Li, K. Tan, H. Viswanathan, Y. Xu, and Y. Yang, "Retransmission $\neq$ repeat: simple retransmission permutation can resolve overlapping channel collisions," in ACM MOBICOM, 2010.

[15] S. Gollakota and D. Katabi, "ZigZag decoding: combating hidden terminals in wireless networks," in ACM SIGCOMM, 2008.

[16] D. Tse and P. Viswanath, Fundamentals of wireless communication. Cambridge university press, 2005.

[17] A. El Gamal and Y.-H. Kim, Network information theory. Cambridge University Press, 2011.

[18] T. M. Cover, R. J. McEliece, and E. C. Posner, "Asynchronous multipleaccess channel capacity," IEEE Transactions on Information Theory, vol. 27, no. 4, pp. 409-413, 1981.

[19] S. Verdu, "The capacity region of the symbol-asynchronous gaussian multiple-access channel," IEEE Transactions on Information Theory, vol. 35, no. 4, pp. 733-751, 1989.

[20] Y. Wu, P. A. Chou, and S. Y. Kung, "Information exchange in wireless networks with network coding and physical-layer broadcast," in Proc. Conference on Information Sciences and Systems (CISS), 2005.

[21] S. Katti, H. Rahul, W. Hu, D. Katabi, M. Médard, and J. Crowcroft, "XORs in the air: Practical wireless network coding," in ACM SIGCOMM, 2006.

[22] T. Li, M. Han, A. Bhartia, L. Qiu, E. Rozner, Y. Zhang, and B. Zarikoff, "CRMA: Collision-resistant multiple access," in ACM MOBICOM, 2011.

[23] G. Cocco, N. Alagha, C. Ibars, and S. Cioni, "Network-coded diversity protocol for collision recovery in slotted ALOHA networks," International Journal of Satellite Communications and Networking, 2013.

[24] M. Khabbazian, F. Kuhn, N. Lynch, M. Médard, and A. ParandehGheibi, "MAC design for analog network coding," in ACM FOMC, 2011.

[25] J. Perry, P. Iannucci, K. Fleming, H. Balakrishnan, and D. Shah, "Spinal codes," in ACM SIGCOMM, 2012.

[26] A. Gudipati and S. Katti, "Strider: Automatic rate adaptation and collision handling," in ACM SIGCOMM, 2011.

[27] H. S. Rahul, S. Kumar, and D. Katabi, "JMB: scaling wireless capacity with user demands," in ACM SIGCOMM, 2012.

[28] H. V. Balan, R. Rogalin, A. Michaloliakos, K. Psounis, and G. Caire, "AirSync: Enabling distributed multiuser MIMO with full spatial multiplexing," IEEE/ACM Transactions on Networking, vol. 6, no. 21, pp. 1681-1695, 2013. 